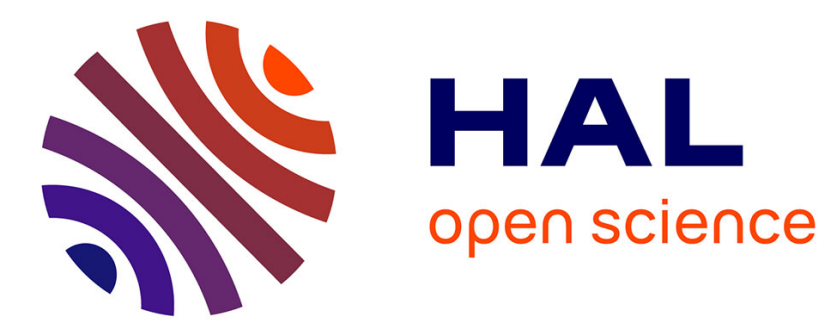

\title{
Nouvelle définition de l'enceinte romaine de Toulouse
} Raphaël de Filippo

\section{To cite this version:}

Raphaël de Filippo. Nouvelle définition de l'enceinte romaine de Toulouse. Gallia - Fouilles et monuments archéologiques en France métropolitaine, 1993, 50, pp.181-204. 10.3406/galia.1993.2937. hal-01912169

\section{HAL Id: hal-01912169 \\ https://hal.science/hal-01912169}

Submitted on 29 Jan 2020

HAL is a multi-disciplinary open access archive for the deposit and dissemination of scientific research documents, whether they are published or not. The documents may come from teaching and research institutions in France or abroad, or from public or private research centers.
L'archive ouverte pluridisciplinaire HAL, est destinée au dépôt et à la diffusion de documents scientifiques de niveau recherche, publiés ou non, émanant des établissements d'enseignement et de recherche français ou étrangers, des laboratoires publics ou privés.

\section{(이) $\$$}

Distributed under a Creative Commons Attribution - NonCommercial - NoDerivatives| 4.0 


\title{
Nouvelle définition de l'enceinte romaine de Toulouse
}

\author{
par Raphaël de FILIPPO*
}

Une opération archéologique de sauvetage réalisée en 1988 sur le site de Larrey à Toulouse permit l'exploration d'importants vestiges de l'enceinte romaine. La conjonction des données dues à la découverte d'un "repentir» dans le tracé de l'ouvrage, à l'analyse stratigraphique des niveaux liés à sa phase de construction et à l'étude archéomagnétique de ses matériaux, conduit à une datation précise de l'édifice. Édifiée vers 30 après J.-G., l'enceinte toulousaine est la seule à être bâtie totalement en briques en Gaule. Si une étude élargie montre que ses précédents se situent en Cisalpine, elle relève d'une conception architectonique unique où s'accordent innovations techniques et rémanences hellénistiques. La nouvelle définition de son enceinte restitue à Toulouse, colonie de droit latin, sa qualité de "cité augustéenne» due peut-être au Princeps lui-même et accomplie sous son successeur Tibère.

A rescue archaeological operation undertaken in 1988 on the Larrey site in Toulouse enabled the survey of important remains of the Roman city wall. The discovery of a "repentance» within the workmanship layout, the stratigraphic analysis of the layers related to its construction and the archaeomagnetic study of its materials gave to the researchers a precise idea about the age of the building. Under construction in 30 A.D., this wall in Toulouse is the only masonry of that kind, in Gaul, to be entirely made of bricks. Although a more extensive study shows that previous examples can be found in Cisalpine, such construction is obviously generaled from a very unique architectonic approach in which technical innovations and hellenistic remanences are closely combined. The new definition of its wall returns to Toulouse - colony placed under the Latin law - its quality of "Augustean city", due perhaps to the Princeps himself, and completed under Tiberius, his successor.

Mots clés : Toulouse, enceinte, construction, brique, Auguste/Tibère.

* Archéologue AFAN, 6, rue Darquier, 31000 Toulouse. 


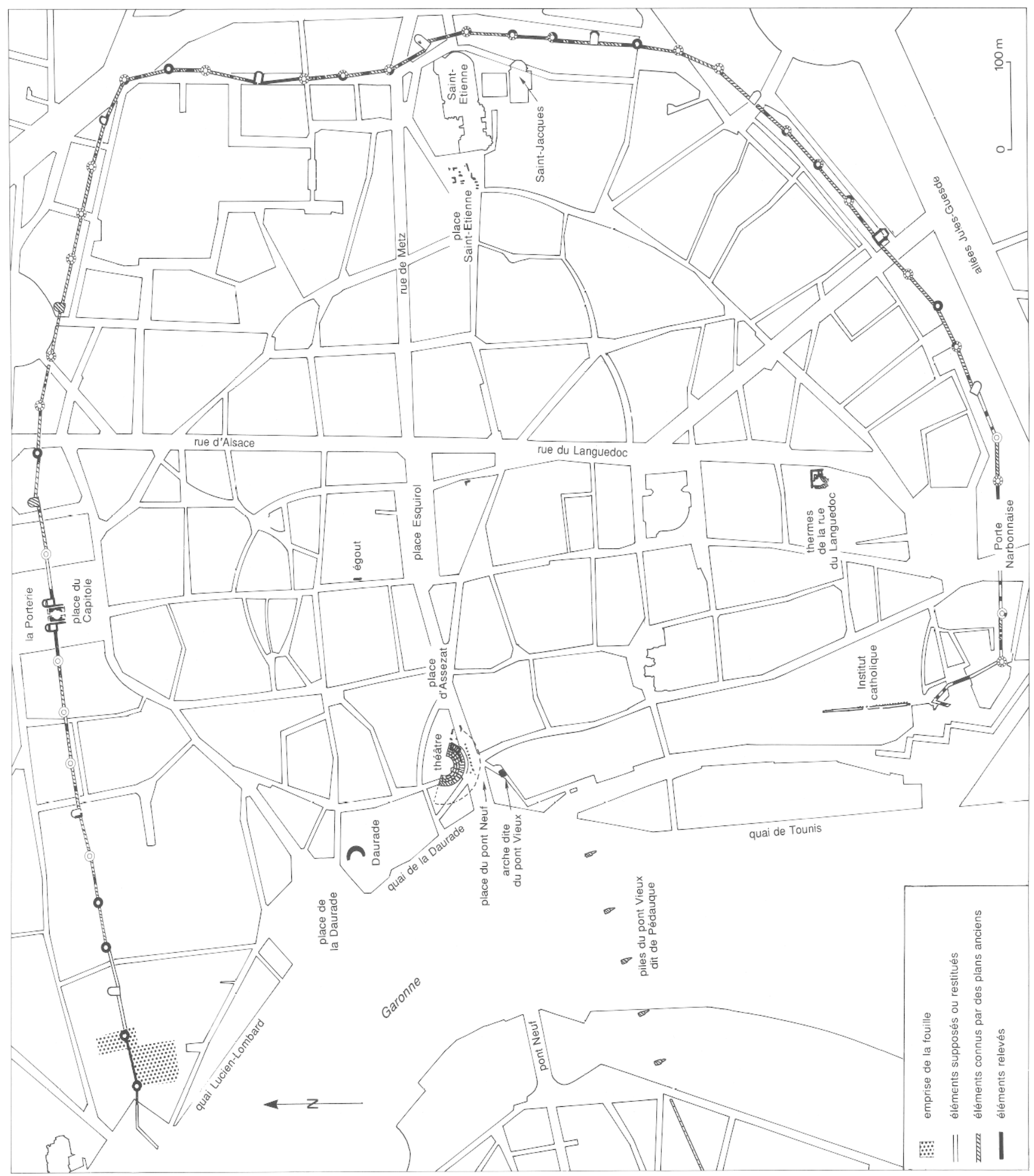




\section{COCTILIBUS MURIS}

Portée sur les plans anciens de la Ville, examinée par les érudits locaux dès le XviI ${ }^{\mathrm{e}} \mathrm{s}$., mise au jour de nombreuses fois ces dernières décennies lors de travaux d'aménagement, repérée systématiquement grâce à des enquêtes minutieuses (Berthe, 1987), l'enceinte gallo-romaine de Toulouse parait être aujourd'hui relativement bien connue, au moins en ce qui concerne sa physionomie générale. Si des lacunes demeurent - pour l'essentiel, à l'endroit des portes Narbonnaise et Saint-Étienne - son tracé et son agencement sont largement restitués (fig. 1). Sa datation est plus controversée. Certes, cela n'est pas une spécificité. Le récent colloque sur Les enceintes augustéennes dans l'Occident romain (1987) a montré que peu d'entre elles sont datées archéologiquement avec précision. Les déterminations proposées s'appuient sur des présomptions qui situent, malgré tout, leur datation dans une marge relativement restreinte. Pour l'enceinte toulousaine, la fourchette, dès l'émergence du débat, est beaucoup trop large: d'Auguste à Ausone (Baccrabère, 1977, p. 106). La plupart des enceintes augustéennes attestées en Gaule sont érigées en Narbonnaise (Goudineau, 1980, p. 244, fig. 166). L'ouvrage toulousain s'en rapproche par ses principales caractéristiques métrologiques d'ensemble (importance du tracé, surface englobée) ou de structure (épaisseur des murs, diamètre des tours). Mais un trait essentiel de son mode de construction empêche cette association: la muraille de Toulouse est faite de briques. Le poète bordelais apporte son propre témoignage oculaire pour célébrer cette particularité : "Coctilibus muris ..." (XIX, Ordo urbium nobilium, vers 98-99). Pour A. Grenier, cette construction ne peut ètre située qu'au Bas-Empire (Grenier, 1931, p. 289), même si cet auteur admet plus tard, mais sans corrélation aucune, que l'emploi d'arases de briques dans l'architecture gallo-romaine apparaît sous le règne d'Hadrien (Grenier, 1958, p. 72-73). Avec raison, M. Labrousse prend cet argument, alors inflexible, pour fixer un terminus a quo à la datation de l'enceinte toulousaine qu'il place finalement au $\mathrm{II}^{\mathrm{e}} \mathrm{s}$., après avoir justement démontré les rapports étroits de ressemblance entretenus entre cette réalisation, isolée en son temps, et celles d'époque augustéenne, alléguant une même intention définie, - "en un siècle où Toulouse a pu acquérir le titre envié de colonie romaine" - (Labrousse, 1968, p. 273-276). Il faut ajouter que la datation de l'accession de Toulouse au titre de colonie attesté par Ptolémée (Géographie, II, $10,6)$ est aussi délicate que celle de l'enceinte
(Labrousse, 1968, p. 491-493). P.-A. Février franchit le pas. Il établit une corrélation directe entre l'édification de l'enceinte toulousaine, dont il retient la datation au $\mathrm{Ir}^{\mathrm{e}} \mathrm{s}$. (les fameuses arases de briques), et l'élévation de la cité au rang de colonie (Février, 1969, p. 280) dues toutes deux, logiquement, à Trajan ou Hadrien. D'ailleurs, depuis A. Grenier, J.-P. Adam n'a pu décaler l'introduction des arases de briques dans l'architecture gallo-romaine que d'un seul empereur (Adam, 1984, p. 154). A l'encontre de l'hypothèse étayée par P.-A. Février, J.-M. Pailler propose un nouveau postulat non moins attrayant. La corrélation entre enceinte et colonie reste admise, mais la démonstration est inverse: deviner quel empereur, présentement Domitien, confèra à la cité des Tolosates son statut et enrichit sa titulature, permet d'attribuer au même souverain l'érection de l'enceinte. La présence des arases n'est plus un problème depuis que cette technique a été reconnue à l'amphithéâtre de Purpan (Toulouse) daté vers le milieu du $\mathrm{I}^{\mathrm{er}} \mathrm{s}$., grâce notamment à l'étude archéomagnétique de ses matériaux (Pailler, 1988, p. 102103). A ces deux précédentes démarches, somme toute conjecturales, s'opposent les travaux de l'abbé Baccrabère fondés exclusivement sur des données archéologiques de terrain, et auxquels il faut rendre justice. La découverte, rue du Rempart SaintÉtienne, de divers niveaux formés au pied de l'enceinte lors de sa construction ou peu après permet, par l'analyse du mobilier collecté, d'avancer la datation de l'édifice au début du $\mathrm{I}^{\mathrm{er}} \mathrm{s}$. (Baccrabère, 1977, p. 37-41). Si l'interprétation de ces niveaux consécutifs à «l'implantation d'un sentier de type militaire longeant le rempart" doit être corrigée, leur rapport stratigraphique avec l'enceinte paraît indéniable. La fouille comparable d'une couche correspondant au niveau de travail pendant sa construction accorde à l'enceinte d'Orange la datation la plus concrète attribuée en Gaule romaine à ce type d'ouvrage (Magdinier, Thollard, 1987, p. 90).

Si la discussion de la datation de l'enceinte de Toulouse engendre encore la polémique, il est une question qui curieusement n'a jamais été abordée. Longtemps, dans l'historiographie du rempart (Berthe, 1987), un détail architectonique a été étroitement lié au problème de la datation : la présence d'un chaînage de briques dans son soubassement en petit appareil (Pailler, 1988, p. 103). L'élévation supérieure réalisée complètement en opus testaceum (Labrousse, 1968, p. 261) n'a jamais paru étonner, il est vrai, dans la "Ville Rose", mais aussi dans une Gaule romaine qui n'a guère connu ce type de maçonnerie (Adam, 1984, p. 154). L'amphithéâtre de 
Purpan et le théâtre antique de Toulouse sont aussi parementés de briques et sont également datés du $1^{\text {er }}$ s. (Cazes, 1988, p. 83). Si à Rome le Castro Pretorio est le premier grand édifice construit en opus testaceum (Adam, 1984, p. 148), les enceintes de Vérone et de Turin (courtines et portes) sont les plus anciens monuments de l'architecture romaine revêtus de briques (Mansuelli, 1971, p. 112-113). Le rempart turinois comporterait aussi des chaînages de briques (Rebecchi, 1987, p. 145). Remarquer et admettre enfin ce rapport de coïncidence semblera évident, mais restera insuffisant. Un autre aspect de l'architecture toulousaine, celte fois spécifique à l'enceinte, demeure encore plus ténébreux. L'originalité de la structure interne des murs de courtine en opus caementicium coulé dans des caissons de briques constitués par les parements eux-mêmes et des murettes transversales (Labrousse, 1968, p. 254) est simplement remarquée. Aucune explication n'a encore été donnée à ce mode constructif si élaboré et tellement particulier à l'enceinte de Toulouse. Son origine sera retrouvée dans l'architecture militaire hellénistique.

\section{L'OPÉRATION ARCHÉOLOGIQUE}

\section{Les perspectives de la recherche}

La plupart des problèmes soulevés par la grande enceinte gallo-romaine de Toulouse sont résolus lors d'une opération archéologique de sauvetage programmé réalisée en 1988. Cette année là se présente enfin l'opportunité d'accéder en un lieu réputé conserver d'importants vestiges du rempart, au nord-ouest de la ville antique, où son parcours se termine aux abords de la Garonne (fig. 1). Bien connue à partir de 1634, l'histoire de l'occupation du site date de l'installation des Sceurs de Notre-Dame, rue du Sac. En 1791, le couvent est déclaré «Bien national» et reçoit comme affectation celle d'hôpital militaire qui prend en 1913 le nom d'Hippolyte Larrey. En 1985, l'hôpital est transféré à Pouvourville, dans la banlieue, et la Ville de Toulouse décide la mise en place d'un programme immobilier et de réhabilitation des anciens bâtiments conventuels. La partie de l'établissement concernée par la fouille correspond au jardin potager des religieuses qui avait été transformé en cour de service, avec adjonction de bâtisses, par les militaires (Grama, 1986).

"... Comme aussi la maison et bâtiment où le jardier habitte qui semble être en deux maisons joignant les vieilles murailles... l'autre partie semble avoir été entièrement une tour...» : ceci est extrait d'un acte de vente, daté du $1^{\text {er }}$ août 1634 , établi entre la veuve Cluzel et les Scours de Notre-Dame qui sans cesse agrandiront leur domaine jusqu'à leur départ forcé. Les mentions anciennes de l'enceinte romaine dans ce secteur de la ville sont dues à la persistance dans la matrice cadastrale de Toulouse de cette limite parcellaire que marque le vieux rempart entre les Capitoulats de la Daurade et de SaintPierre. Le cadastre Jouvin de Rochefort (1680), le plan Saget (1750) et le cadastre Grand Voinet (17801808) soulignent que le bornage nord du clos de l'établissement conventuel se confond avec les vieilles murailles de la ville, en précisant l'emplacement des tours (Rivals et alii, 1972). Le surnom même donné aux religieuses, "les Dames du Sac", vient de ce que l'entrée principale du couvent se situait près du culde-sac d'une rue barrée, encore à leur époque, par le franchissement de l'enceinte antique (Grama, 1986, pl. III).

Cette permanence de la connaissance du tracé général de l'enceinte dans la ville moderne et plus précisément la conviction qu'à l'hôpital Larrey subsistaient encore d'importants vestiges conduisent $\mathrm{P}$. Fort à effectuer sur les lieux, dès 1948, les premières observations véritables. L'inspection scrupuleuse de la courtine le mène à la découverte de la structure à caissons de coffrage des parties hautes du mur, seulement connue par une mention de l'abbé Magi au $\mathrm{XVII}^{\mathrm{H}} \mathrm{s}$. et reprise par Dumège un siècle plus tard. $\mathrm{P}$. Fort retrouvera la même technique sur le rempart de Garonne à l'Institut catholique de Toulouse (Labrousse, 1968, p. 261).

Toutes les enquêtes de repérage des vestiges de l'enceinte dans la ville actuelle passent par la visite des caves car c'est seulement dans les sous-sols des maisons adossées à la muraille que le soubassement en moellons calcaires peut être encore visible; les parties hautes, là où elles subsistent, sont masquées par des revêtements posés depuis le Moyen Age jusqu'à nos jours. Cett.e démarche systématique conduite par l'abbé Baccrabère sur l'ensemble du tracé de l'ouvrage aboutit à un inventaire précis des vestiges de l'hôpital Larrey : deux tours rondes qui flanquent une courtine dont le soubassement paraît être intact et dont le mur conserve une hauteur importante devraient être dégagées lors de l'opération archéologique (Baccrabère, 1977, p. 44). L'accession à cet ensemble considérable permet d'envisager une analyse architectonique complète du rempart et d'appréhender les problèmes de datation. Cette détermination peut être atteinte par l'examen st.ratigraphique des niveaux archéologiques accumulés au pied de la muraille. A l'hôpital Larrey, côté 
intra-muros, c'est un terrain de $5000 \mathrm{~m}^{2}$ qui devient accessible aux archéologues.

\section{L'exploration des vestiges de l'enceinte}

La première intervention sur le site consiste à démolir les bâtiments construits par les militaires sur l'ancien potager des religieuses. Le grand édifice réservé à l'accueil des malades et dont le mur nord correspond à celui de l'enceinte est rasé au niveau du sol. Insérés entre ce bâtiment et celui de l'horloge, d'origine conventuelle, la buanderie et le salon de coiffure sont également détruits. Leur démolition permet le dégagement d'une tour et l'accès au seul espace extra-muros disponible pour la fouille. Après un conditionnement, pour des raisons de sécurité, des murs dégagés, les vestiges apparaissent conformes aux observations effectuées par le passé. La tour est, avec l'intégralité de son plan circulaire, est conservée à un niveau inférieur au sol actuel (143 m NGF). La courtine s'élève à $4 \mathrm{~m}$ au-dessus. La tour ouest garde une hauteur identique à celle de la courtine, mais seulement sur un quart de sa circonférence. La mise au jour des parties basses de l'enceinte, situées sous le sol contemporain, se fait au fur et à mesure de l'avancement de la fouille des niveaux archéologiques constitués sur le site des origines de son occupation jusqu'à nos jours.
La tour est ne subsiste que sur $1 \mathrm{~m}$ de hauteur (fig. 2). Le parement d'origine, très lacunaire, est remplacé par des reprises d'époques médiévale et moderne. Même les fondations, côté campagne, sont largement reprises en sous-œuvre par un rhabillage de briques. Finalement, le mur de courtine, depuis ses fondations, atteint encore une hauteur de $6,50 \mathrm{~m}$. Le creusement de nombreuses fosses-dépotoirs médiévales et l'insertion des fondations de bâtisses d'époque moderne ont entamé en plusieurs endroits le parement de base en petit appareil, surtout dans le secteur est. Côté ouest, sa conservation est totale jusqu'à la tour comprise. Cette dernière porte encore, sur $1 \mathrm{~m}$ de haut, son parement d'origine en briques qui, côté ville, a pratiquement disparu (fig. 3). Au contraire, sur l'autre face, le parement supérieur en briques, découvert sur plusieurs mètres, semble être en état sur toute la hauteur du mur. La disparition du parement sur la totalité de la paroi mise à nu a pour bénéfice de laisser apparaître, pour la première fois de façon aussi évidente, la structure du blocage si particulière à l'enceinte toulousaine.

Une inspection minutieuse des deux tours et de la courtine permet de dissocier les reprises nombreuses des structures originales. C'est ainsi qu'il apparaît que les tours, en élévation, ont une ossature interne bâtie uniquement de briques, sans blocage en opus caementicium. Le nettoyage (environ $200 \mathrm{~m}^{2}$ ) du blocage de la courtine rend parfaitement distinctes

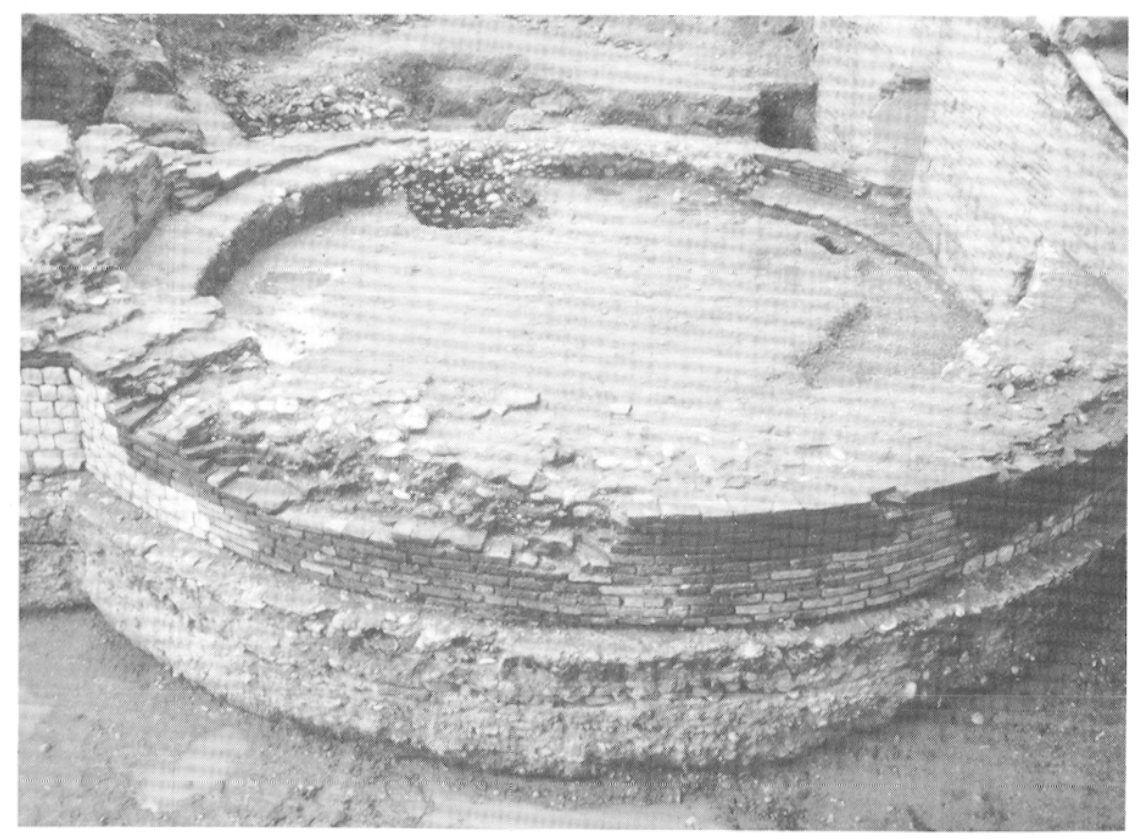

Fig. 2 Tour est, vue côté intra-muros. 


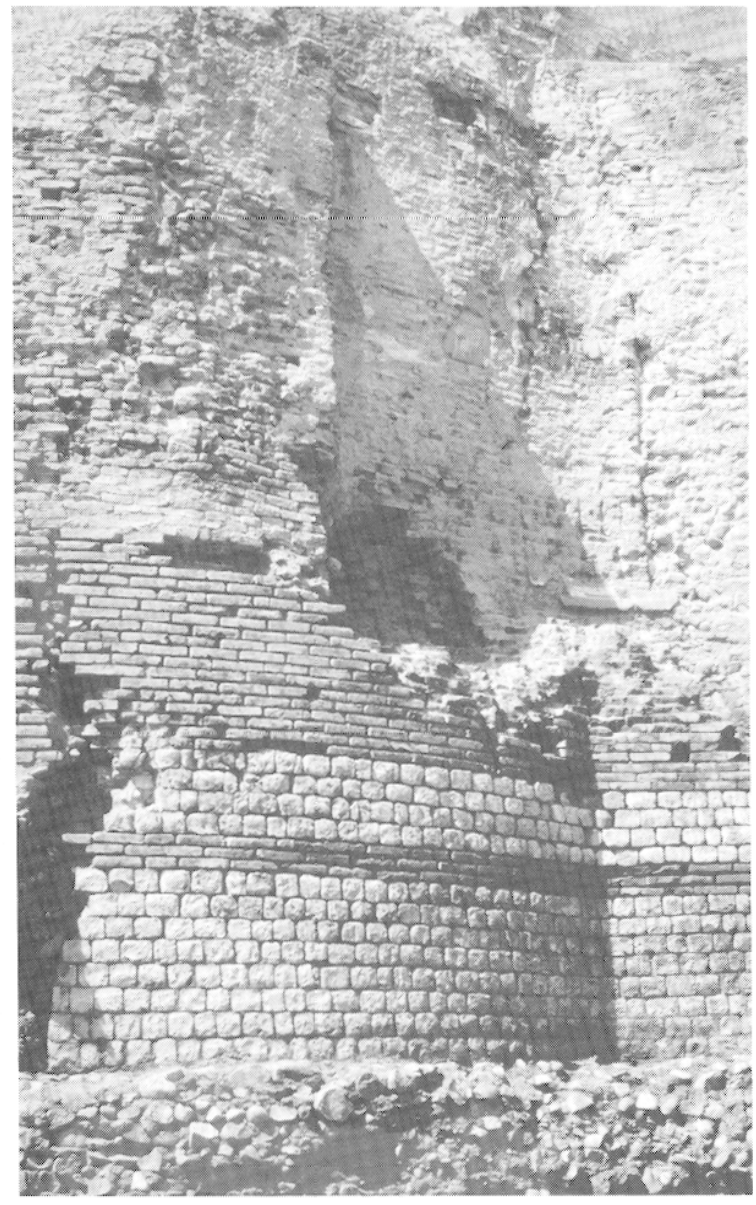

Fig. 3 - Tour ouest, vue côté intra-muros. ses murettes en briques et surtout les limites de chaque banchée (fig. 4). A l'issue de la fouille, une tranchée est aménagée tout le long de l'enceinte pour faciliter l'examen complet des fondations jusqu'à leur base. Cette observation entraîne la découverte d'un fragment de céramique sigillée scellé dans le mortier. Des échantillons de mortier sont prélevés en plusieurs points et des lots de briques sont pris sur les parements, les chainnages et les murettes du blocage. Ce démontage permet la découverte des premières estampilles de briquetier, inédites, trouvées en situation dans le rempart. Cette investigation sur les vestiges de l'enceinte s'achève par un relevé graphique intégral (plan, coupes, élévations) et une couverture photographique globale et détaillée.

\section{Une rectification dans le tracé de l'enceinte}

Lors de la fouille des couches archéologiques constituées le long de l'enceinte, à 32,40 m de la tour est, au niveau de la semelle de fondation de la courtine, est mise au jour une structure qui apparait, de prime abord, comme aberrante. Son identification se fera au fur et à mesure de son dégagement. C'est un massif en opus caementicium, installé en tranchée, qui prolonge, sans interruption de coulée, les fondations de la courtine (fig. 5). Au départ, son plan décrit un arc de cercle similaire au tracé intra-muros d'une tour. De la retombée de cette courbe, part un segment rectiligne, long de $9,50 \mathrm{~m}$, et dont la largeur

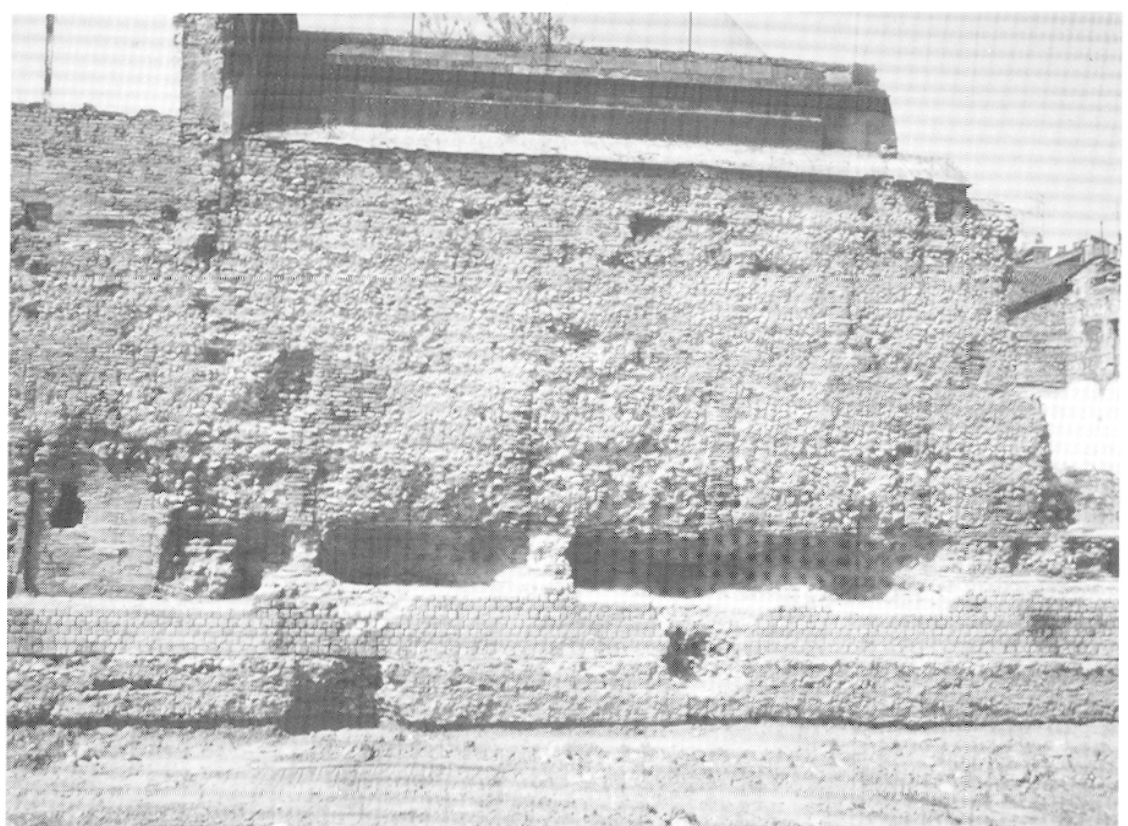

Fig. 4 -

Paroi, côté intra-muros, de la courtine où apparaissent les murettes de briques de la structure du blocage mise à nu. 


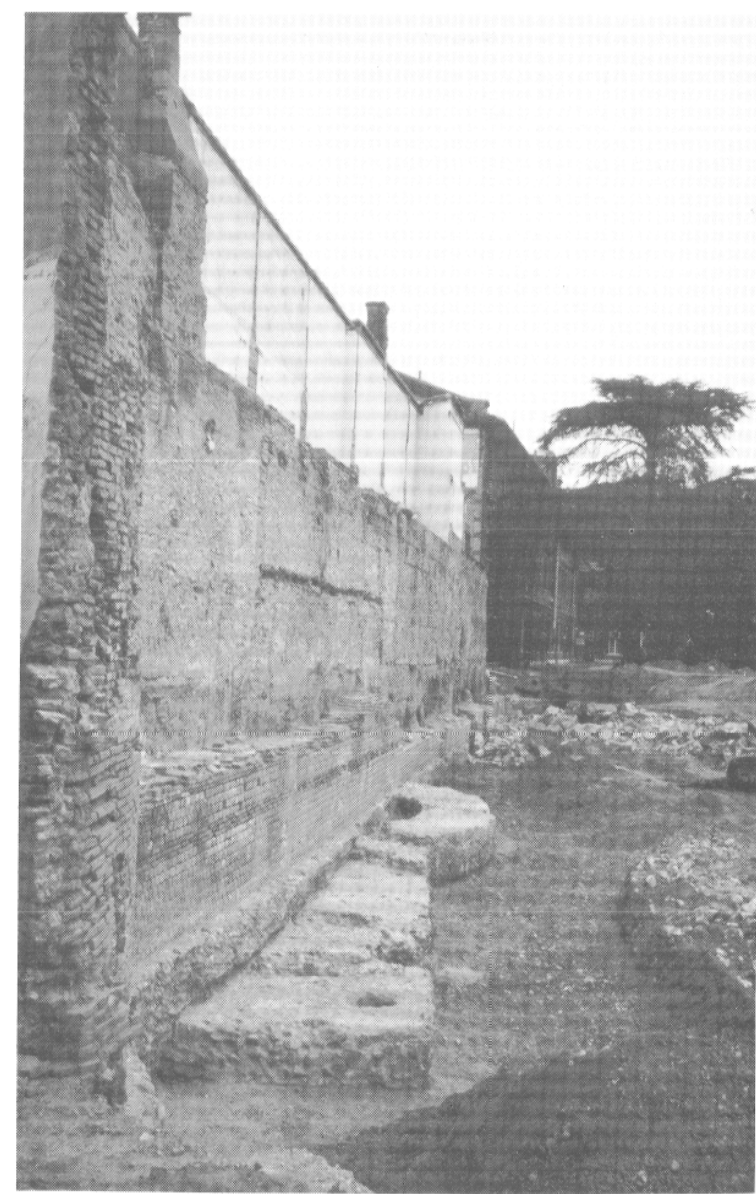

Fig. 5 - Les fondations de la tour et du départ de la courtine abandonnées après correction du tracé de l'enceinte.

égale celle des fondations de la courtine. Dans le prolongement de cette structure, la fouille a mis en évidence l'existence d'une tranchée qui continue le tracé de l'ensemble. Elle est désobstruée seulement sur une longueur de $5,50 \mathrm{~m}$, jusqu'aux limites du chantier au-delà desquelles elle s'étend encore. Cet ensemble témoigne d'un projet initial d'une tour et du départ d'une nouvelle courtine abandonné alors que les fondations sont en cours de réalisation.

Les fondations de la tour sont achevées. C'est la superposition de deux niveaux de coulée de même importance qui leur donne une épaisseur totale de $0,90 \mathrm{~m}$. Le premier niveau est la continuation de celui des fondations de la courtine précédente et audelà du tracé de la tour c'est toujours la même banchée qui entame le départ de la courtine suivante. Celle-ci s'interrompt sur un front en biseau dont la face plane est obtenue par le sciage des galets du garnissage de l'opus caementicium. Le second niveau, s'il parachève les fondations de la tour, n'a pas été terminé sur le tronçon de courtine où il n'atteint qu'une épaisseur de $0,25 \mathrm{~m}$ au lieu des $0,45 \mathrm{~m}$ définitifs. Cette rupture intervient brutalement au contact de la tour et de la courtine.

La tranchée ouverte en avant sur une distance qui demeurera inconnue devait recevoir l'extension de ces fondations. L'abandon du projet induit le comblement de la tranchée qui s'effectue après un certain laps de temps marqué par un effondrement partiel de ses parois. Le remblayage se fait avec les graviers et les limons extraits lors du creusement même de la tranchée. A ces matières sont mêlés de nombreux fragments d'amphores du type Dressel 1 d'importation italique et deux fragments de céramiques sigillées arétines (Ritt. 5 et Drag. 11) attribuables aux deux premières décennies de notre ère. La dénivellation qui existe entre la tour et la courtine est également rattrapée par un comblement de cailloutis et de limons dans lesquels sera trouvé un demi-as de Nîmes, deuxième émission (fin $\mathrm{I}^{\mathrm{er}} \mathrm{s}$. avant/début $\mathrm{I}^{\mathrm{er}} \mathrm{s}$. après $\mathrm{J}$.-C.). Suite au recouvrement de ces fondations définitivement délaissées, le tracé de la courtine déjà réalisée est repris sur le même axe sur une longueur supplémentaire de $19 \mathrm{~m}$, jusqu'à la prochaine tour effectivement construite (fig. 6 et 7).

La découverte de cette esquisse d'une tour et d'une nouvelle courtine est très instructive sur les méthodes d'implantation des fondations ainsi que sur les phases du déroulement du chantier de construction de l'enceinte. Cette rectification ne relate pas une simple erreur de maçons. Elle prouve immanquablement l'hésitation des architectes et topographes sur la façon de terminer l'enceinte aux abords de la Garonne. Leur incertitude est si flagrante qu'ils modifient leur projet, et renoncent ainsi à des travaux déjà bien avancés. Enfin, l'intérêt majeur de cette découverte imprévue est de livrer d'importants éléments pour la datation de l'enceinte.

\section{Les données stratigraphiques}

L'implantation de l'enceinte sur le site de l'hôpital Larrey s'opère sur un terrain libre de toute occupation. Le dernier établissement remonte à la fin de l'Age du Bronze et n'est plus perceptible, à l'époque romaine, dans la topographie. Le sol est constitué par les alluvions de la Garonne : un limon très sableux, d'une épaisseur moyenne de $0,40 \mathrm{~m}$, qui surmonte la terrasse caillouteuse du fleuve sur laquelle s'est édifiée la ville romaine (fig. 8).

Comme le terrain est naturellement en pente vers le fleuve, c'est, semble-t-il, sur une légère ligne 


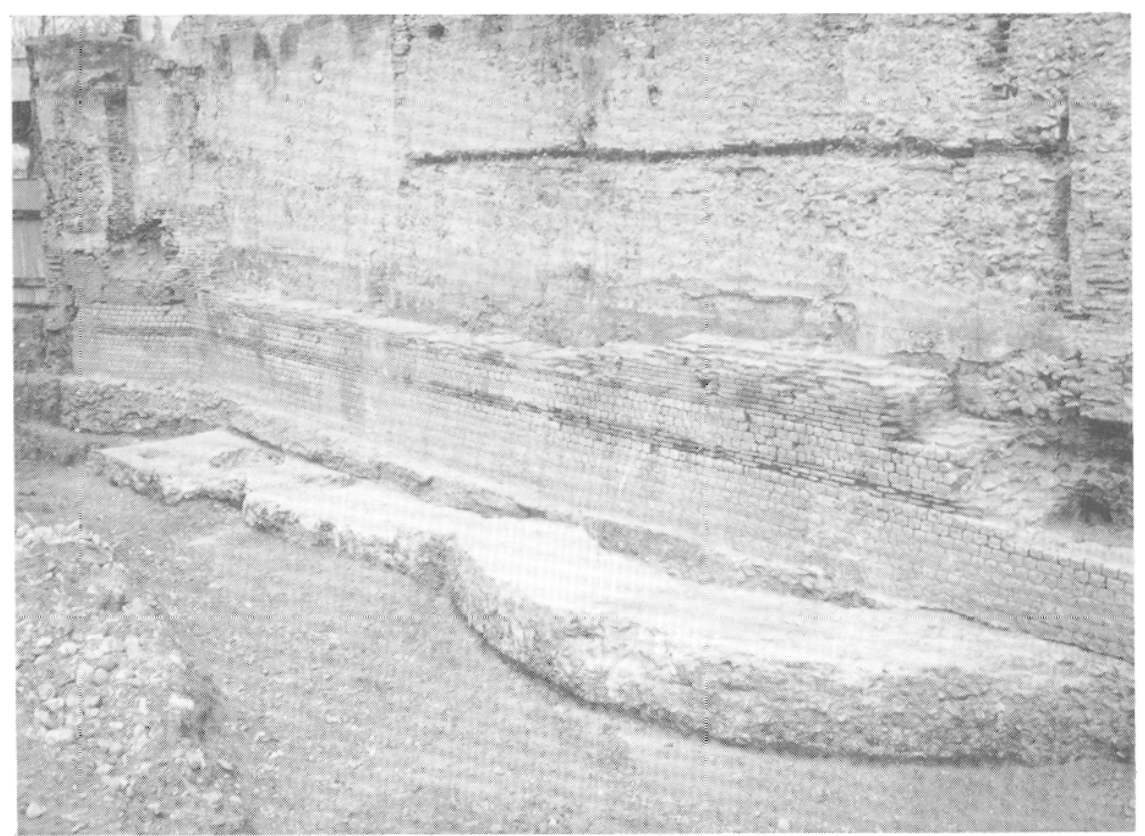

Fig. 6 - Les fondations abandonnées et la réalisation effective.

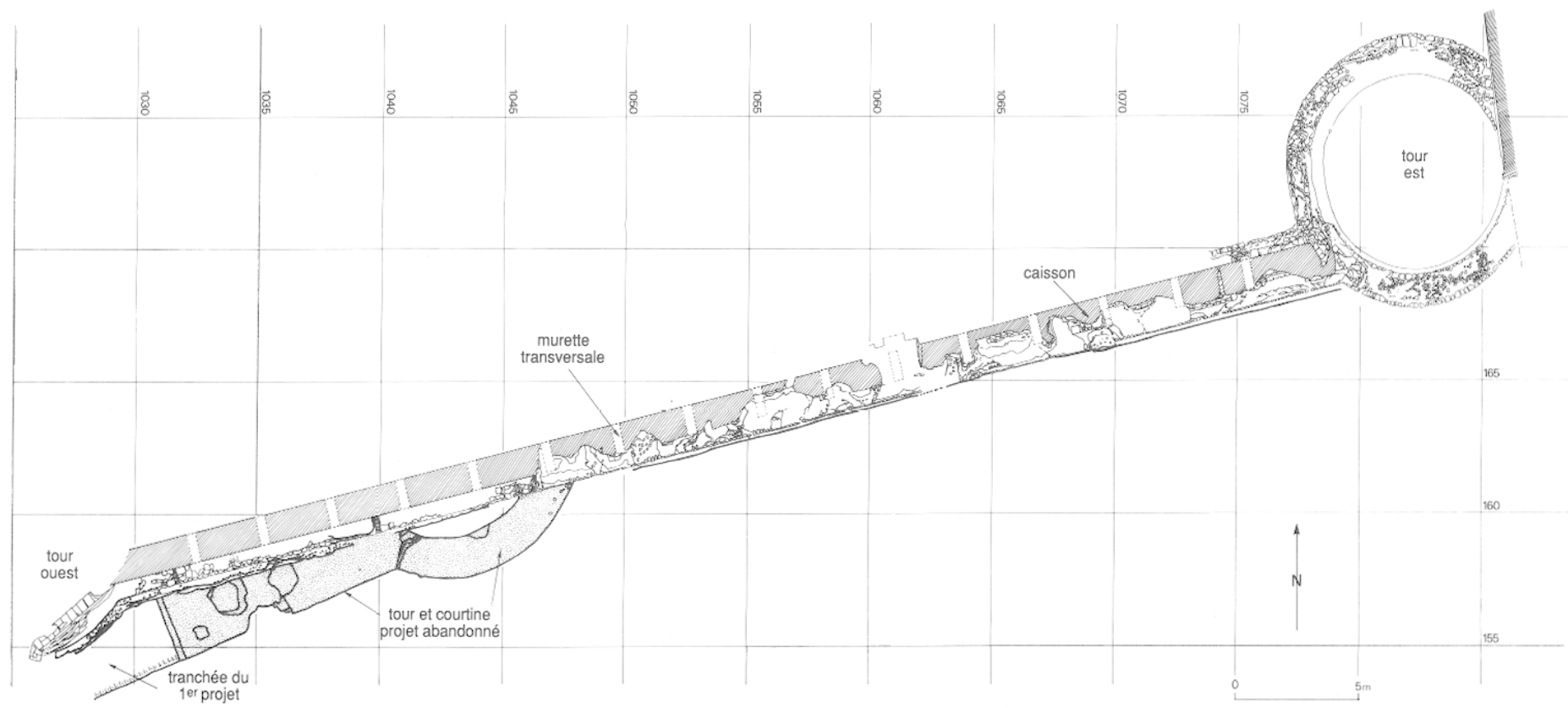

Fig. 7 - Plan des vestiges de l'enceinte à I'hôpital Larrey dégagés lors de l'opération archéologique. 


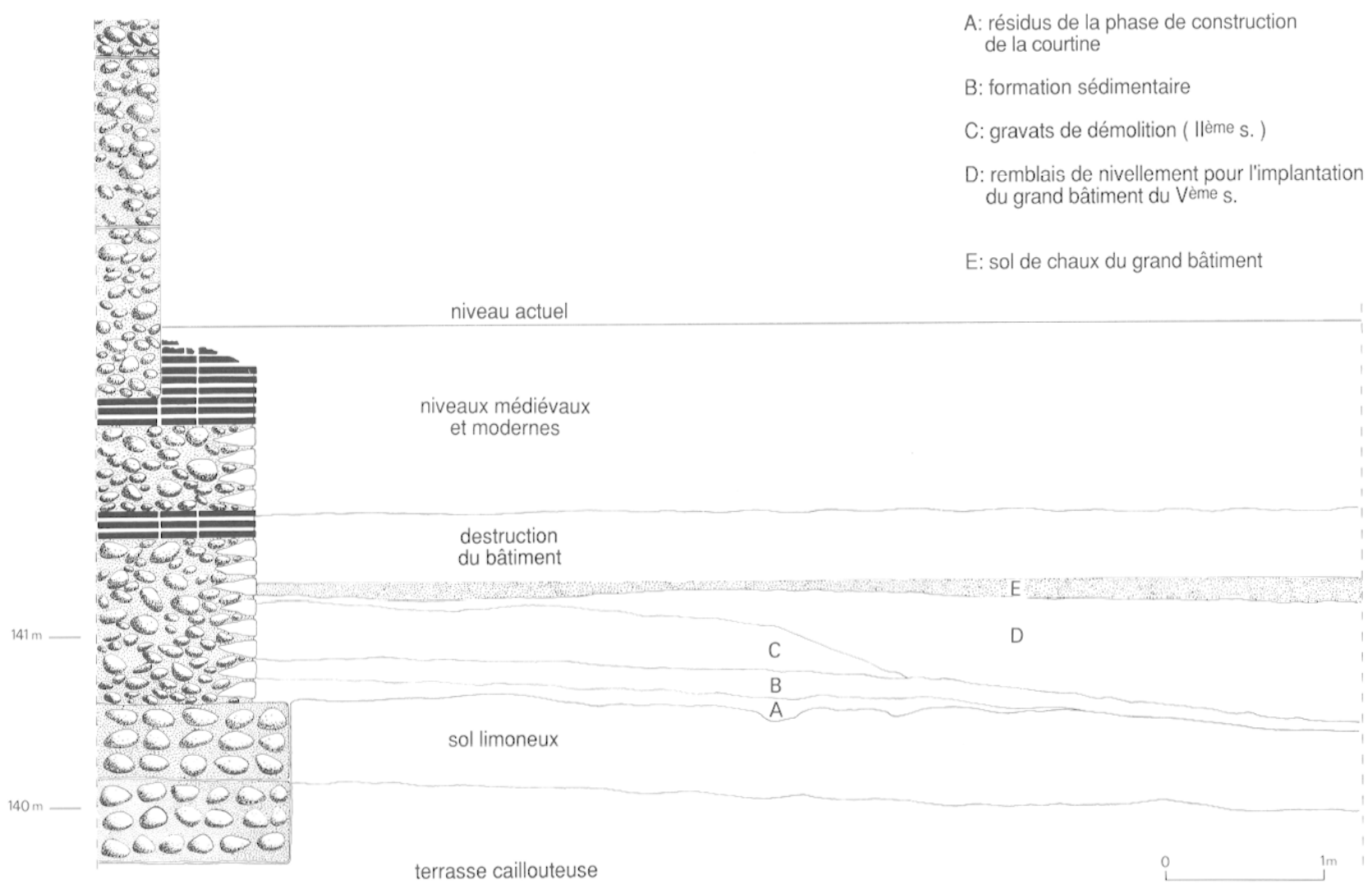

Fig. 8- Coupe stratigraphique des niveaux accumulés au pied de la courtine, pendant et après sa construction : axe nord-sud 1055 .

de crête, à 140,60 m NGF (altitude moyenne), que sont installées les fondations de l'enceinte. La péripétie qui conduit à la correction du tracé de la courtine apporte les premiers éléments probants pour déterminer la datation de l'enceinte. La solution de ce problème est confortée par le repérage dans la stratigraphie des niveaux laissés sur le sol pendant cette phase de travaux. De la tour avortée à la tour ouest, au-dessus des remblais amenés pour enterrer les fondations du premier projet s'est formée une épaisse et solide croûte blanchâtre constituée d'un lit de chaux auquel se sont agglomérés des cailloutis et de petits fragments de briques. Au pied de l'enceinte sur laquelle il s'appuie en surface et à hauteur de la deuxième assise du parement, son épaisseur dépasse $0,20 \mathrm{~m}$. Au fur et à mesure de son éloignement du rempart, elle diminue progressivement, ainsi que sa teneur en chaux, pour disparaître totalement à une distance de 4,30 $\mathrm{m}$. Un demi-as de Nîmes de la première émission et de minuscules fragments de céramiques sigillées de Montans ont été pris dans la chaux. L'étude des tessons fournit une fourchette chronologique définie entre 30 et 80 après J.-C. Contemporaine de la phase de construction de l'enceinte, cette couche correspond à une aire de gâchage du mortier nécessaire à la maçonnerie de l'élévation ; cela explique qu'elle recouvre l'empattement des fondations du soubassement. Au mieux postérieure, elle livre néanmoins de sérieux indices pour fixer un terminus ante quem à la date de construction. Sa localisation au-dessus des fondations délaissécs nc pcut pas être fortuite. Elle stabilise aussi la surface d'un terrain remanié.

Dans une position stratigraphique et topographique similaire à celle de l'aire de chaux indurée, une autre couche s'est formée au pied de l'enceinte pendant la durée des travaux. Elle apparaît comme un semis de graviers où s'agglomèrent des galets, des fragments de briques et des éclats de calcaire identique à celui des moellons du soubassement. Cette couche cache la première assise de l'élévation (140,72 m NGF). En s'écartant de l'enceinte, elle devient scnsiblement plus diffuse pour s'effacer complètement au bout de $6 \mathrm{~m}$ (fig. 9 et $8 \mathrm{~A}$ ). Le rap- 


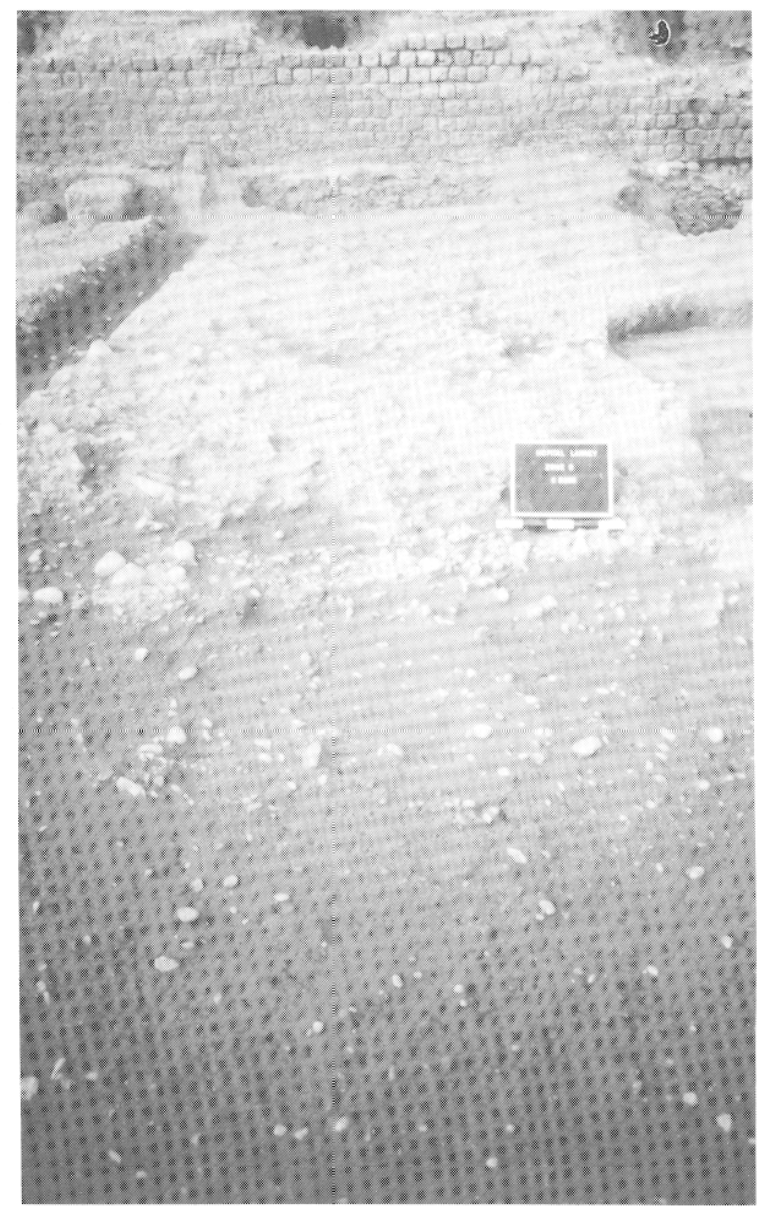

Fig. $9-$ Au pied de la courtine, couche résiduelle de gravats due à la phase de construction de l'enceinte.

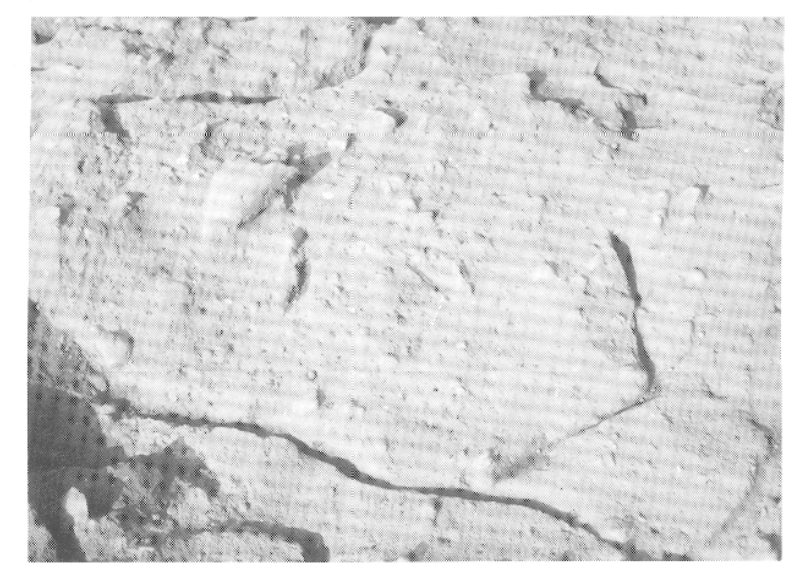

Fig. 10 - Formation sédimentaire, parcourue de racines en négatif, qui recouvre les résidus de la phase de construction (cf. fig. 8). port d'équivalence établi dans le diagramme stratigraphique entre cette couche, résidu d'une zone de travail, et l'aire de chaux se vérifie par l'étude de son mobilier (céramiques sigillées de Montans et de La Graufesenque) qui prouve leur concordance chronologique en permettant de resserrer la fourchette à «30-60 après J.-C.». La validité de ces nouveaux éléments de datation est assurée par la certitude que ces niveaux n'ont pas été perturbés après leur constitution. A la suite de l'achèvement de l'enceinte, le site ne connait pas une occupation dense immédiate. Un seul édifice est implanté à proximité de la tour ouest, dans la seconde moitié du $1^{\text {er }}$ s. $\Lambda u$ pied de l'enceinte, où un puits est aussitôt bâti, une lente sédimentation recouvre finalement les vestiges amassés sur le sol pendant les travaux. La formation de ce nouveau sol limoneux atteint une épaịsseur de $0,10 \mathrm{~m}$ (surface à $140,82 \mathrm{~m} \mathrm{NGF}$ ), à hauteur de la deuxième assise de l'élévation (fig. 8B). De nombreuses racines rampantes, très étalées, retrouvées par l'empreinte négative laissée par leur pourrissement (leur diamètre peut atteindre $0,10 \mathrm{~m}$ ), parcourent le limon (fig. 10). Leur présence certifie la formation progressive de la sédimentation et la permanence de ce nouveau sol. Sa surface correspond ensuite à un niveau de circulation marqué par le sillon d'une ornière et l'implantation d'une base de pilier en maçonnerie de briques. Le mobilier céramique contenu dans le limon donne des datations qui s'étalent du milieu du $\mathrm{I}^{\mathrm{er}} \mathrm{s}$. à la seconde moitié $\mathrm{du}$ ${ }_{I^{\mathrm{e}}}^{\mathrm{e}} \mathrm{s}$., avec une forte prépondérance de la fin du $\mathrm{I}^{\mathrm{er}} \mathrm{s}$. qui coïncide avec l'occupation, à la même époque, des structures voisines de la tour ouest. Il faut rapprocher de cette occupation l'utilisation du puits et aussi la plantation d'arbustes qui favorisent la formation sédimentaire. Trois monnaies accompagnent la céramique : un as de Claude ou une imitation (après 46), un as d'Hadrien (125-163), un as de Faustine (161-176). Le comblement d'un drain aménagé dans le limon livre un sesterce de Marc-Aurèle (162163). Le limon est enfin recouvert de gravats qui proviennent sans doute de la démolition des bâtisses situées devant la tour ouest. De l'amas de briques et de tuiles est issu un dupondius de Commode (181185), (fig. 8C).

\section{L'étude archéomagnétique}

Deux lots de briques prélevés sur l'enceinte ont fait l'objet d'une étude archéomagnétique, effectuée par L. Goulpeau et P. Lanos, au Laboratoire d'archéométrie, département de physique cristalline de l'Iniversité de Rennes (Goulpeau, 1990). I e premier lot de 100 briques (H.L., R.-1) provient du démon- 
tage complet d'une murette transversale d'un caisson de coffrage du deuxième étage, à l'extrémité est de la courtine, près de la tour. Le deuxième prélèvement comprenant 97 échantillons (H.L., R.-2) vient du chaînage encastré dans le soubassement en petit appareil, aux abords de la même tour. La provenance de ces deux lots, dégagés de la masse interne du mur en opus caementicium, préférés à des prélèvements sur les parements, leur assure une garantie d'origine incontestable. L'exploitation des résultats concernant les aimantations rémanentes, mesurées sur chaque échantillon au magnétomètre-spinner, conduit aux dates suivantes:

$$
\begin{aligned}
& \text { H.L., R.- } 1=\text { de } 0 \text { à } 40 \text { après J.-C. } \\
& \text { H.L., R.- } 2=\text { de } 0 \text { à } 30 \text { après J.-C. }
\end{aligned}
$$

L'écart d'une dizaine d'années entre les deux fourchettes chronologiques proposées répercute seulement de légères variations entre les diagrammes archéomagnétiques des deux lots qui présentent dans l'ensemble une remarquable homogénéité, entre eux ou séparément. Les configurations générales des deux lots sont similaires, ce qui indique, avec une forte probabilité, une cuisson dans des fours d'orientation identique (peut-être même un four unique), avec des pratiques technologiques semblables quant aux modalités de chargement du four (briques cuites sur la tranche) par une même équipe d'artisans-tuiliers ou une même tradition artisanale locale (Goulpeau, 1990, p. 1-6).

Si le premier lot (H.L., R.-1) appartient au blocage supérieur et est donc installé obligatoirement après le deuxième lot (H.L., R.-2) issu du soubassement, tous deux proviennent du même secteur $\mathrm{du}$ mur de courtine. L'intervalle entre leur mise en place ne peut être que court. Toutes les briques façonnées sur un module unique sortent d'un même atelier. Chaque lot contient plusieurs briques portant la même estampille anépigraphe : une boucle qui représente, sans doute, la première lettre de l'alphabet grec. Un troisième lot de 111 briques (H.L., P.) démontées du puits placé près de l'enceinte a également fait l'objet d'une analyse. Les résultats conduisent à une datation centrée autour de 40 après J.-C. et inscrite dans l'intervalle " $20-60$ après $\mathbf{J}$.-C.» (Goulpeau, 1990, p. 6-9).

\section{La datation de l'enceinte}

Tout d'abord, s'impose une observation essentielle rendue manifeste non seulement par l'examen des niveaux archéologiques étendus au pied de l'enceinte, mais aussi par les résultats acquis sur l'ensemble du site : la fouille n'a pas révélé la moindre trace d'une "présence gallo-romaine» antérieure à l'édification de l'enceinte. Ainsi, le mobilier trouvé dans les niveaux liés à la phase de construction (tranchée de fondation, aires de travail) n'est pas résiduel. Son apport est à associer à l'implantation du chantier. Cette donnée est vérifiée par le rapport de coïncidence établi entre la surface d'empiétement du chantier et la surface de répartition de ce même mobilier sur le site. As augustéens et céramiques sigillées italiques n'ont pas été retrouvés en dehors des zones de travail. Ils proviennent essentiellement du remblayage des fondations délaissées et fournissent les éléments de datation les plus anciens. Les céramiques sigillées du Sud de la Gaule (La Graufesenque, et surtout Montans) ont été recueillies exclusivement parmi les gravats du chantier, mêlées à la formation sédimentaire qui les recouvre ou dans l'environnement immédiat de l'édifice bâti près de la tour ouest. La chronologie des monnaies associées à chacun de ces niveaux successifs corrobore leur rapport stratigraphique déjà démontré par l'étude du mobilier céramique. Cela fixe un terminus ante quem pour la construction de l'enceinte à 50 après J.-C. Le fragment de céramique sigillée de Montans (forme Drag. 29 à godrons : 30-40 après J.-C.), pris dans les fondations de la courtine proche de la tour est, provient d'un segment coulé avant la réalisation des fondations remblayées. Ce tesson ne détermine pas seulement un terminus post quem indiscutable. En s'intégrant à la fourchette donnée par l'analyse de céramiques ramassées sur les aires de travail, il livre la datation de l'enceinte qui se situe, grâce à la conjonction des études archéomagnétiques, très près de 30 après $\mathbf{J}$.-C.

\section{L'histoire du site relatée par la fouille}

La seule occupation antérieure à l'implantation de l'enceinte sur le site date de la fin de l'Age du Bronze/début de l'Age du Fer. Elle est attestée par la présence de structures (foyer, trous de poteaux) accompagnées de fragments de céramiques dont les formes (plats tronconiques, gobelets, urnes, jarres) et les décors (peignés, incisés, impressionnés, digités) sont bien caractéristiques (Porra, 1990).

Durant les trois premiers siècles de notre ère, le terrain est peu bâti. Seul un ensemble est implanté en avant de la tour ouest dans la seconde moitié du $\mathrm{I}^{\text {er }} \mathrm{s}$. Situé en marge des limites du chantier de fouille et fortement arasé, il ne peut lui être attribué une fonction précise. Ailleurs, sur un vaste espace destiné peut-être à des cultures vivrières, il n'a été repéré que deux puits.

$\mathrm{Au}$ IV $^{\mathrm{e}}$ s., un atelier de potier occupe le site. II produit des dérivés de sigillées à couverte orangée et 
décor estampé (découverte d'un poinçon) et des lampes (découverte des moules). Au v $\mathrm{v}^{\mathrm{e}} \mathrm{s}$., les installations artisanales sont arasées pour permettre l'implantation d'un bâtiment aux dimensions monumentales construit en appui sur le rempart. Sa situation dans la ville, l'importance de son extension et l'originalité de son plan (grandes exèdres opposées, longs couloirs latéraux) qui s'articule en une parfaite symétrie, donnent à cet ensemble un caractère particulier en un siècle où Toulouse est capitale du royaume wisigothique (fig. 11).

Après son abandon, le bâtiment est "démonté» pour la récupération de ses matériaux, notamment les briques. Il faudra attendre le Bas Moyen Age pour qu'une nouvelle zone d'habitat se développe aux abords de la rue des Blanchers et de son impasse (Catalo, 1990). Le reste de l'espace, en arrière-cour, accueille une centaine de fosses-dépotoirs (de Filippo, 1990). Leur fouille livrera une importante quantité de mobilier dans laquelle on remarquera surtout l'apparition d'une verrerie originale (Reverdy, 1990) et la présence de céramiques de Saintonge (de Filippo, 1990).

Les anciens plans cadastraux montrent que les limites parcellaires du secteur étudié ont été établies sur les grands axes du bâliment de l' $\Lambda$ ntiquité tardive et sur le tracé de l'enceinte. C'est en 1700 que les religieuses, pour la création du jardin, achètent à François d'Assézat la parcelle dont la surface correspond à celle de l'opération archéologique.

\section{LA CONSTRUCTION DE L'ENCEINTE}

\section{Les fondations}

Elles sont installées dans une tranchée à fond plat et aux rives verticales, large de $2,80 \mathrm{~m}$ et d une profondeur moyenne de $0,90 \mathrm{~m}$. La tranchée atteint les alluvions caillouteuses de la Garonne. Le massif de fondation en opus caementicium a été coulé en deux niveaux nettement distincts d'épaisseur égale. Le niveau inférieur correspond à l'épaisseur des graves entamées par le creusement. Le niveau supérieur est installé dans les alluvions limoneuses. Chaque niveau, coulé par segments qui se terminent par un front en biseau, est constitué de trois lits de gros galets séparés par d'épaisses couches de mortier de chaux. Les galets en bout de segment sont taillés pour donner une surface plane au biseau. Ce traitement particulier devait assurer sans doute une sorte de joint de dilatation. I'orientation du biseau montre que la progression des travaux se fit, dans ce secteur, en avançant vers la Garonne. La surface plane de la semelle coïncide avec la surface du sol à partir duquel est creusée la tranchée. Cette observation est essentielle pour l'analyse stratigraphique des niveaux archéologiques qui s'appuient à la base de l'enceinte. I.es fondations suivent les variations altimétriques naturelles du site (de 140,70 m à 140,55 m NGF). Le creusement de la tranchée, pour maintenir

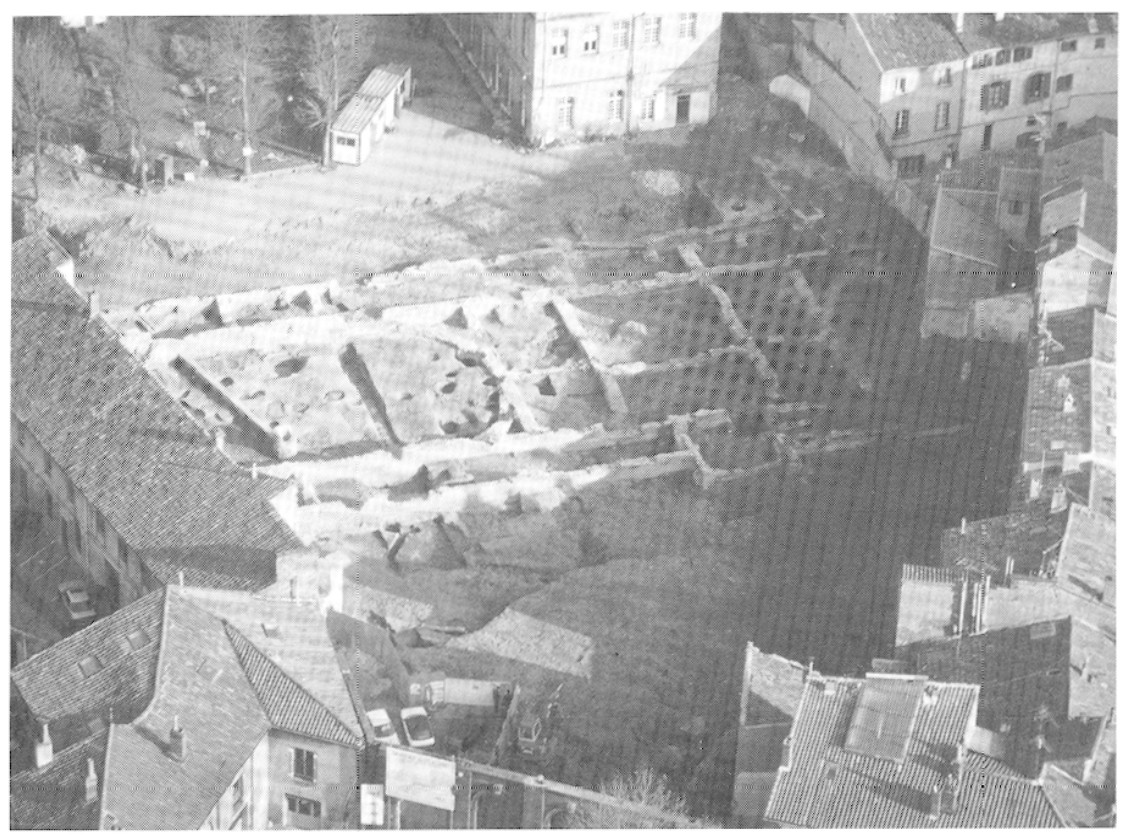

Fig. 11 - Vue aérienne du bâtiment du $v^{\mathrm{e}} \mathrm{s}$. 


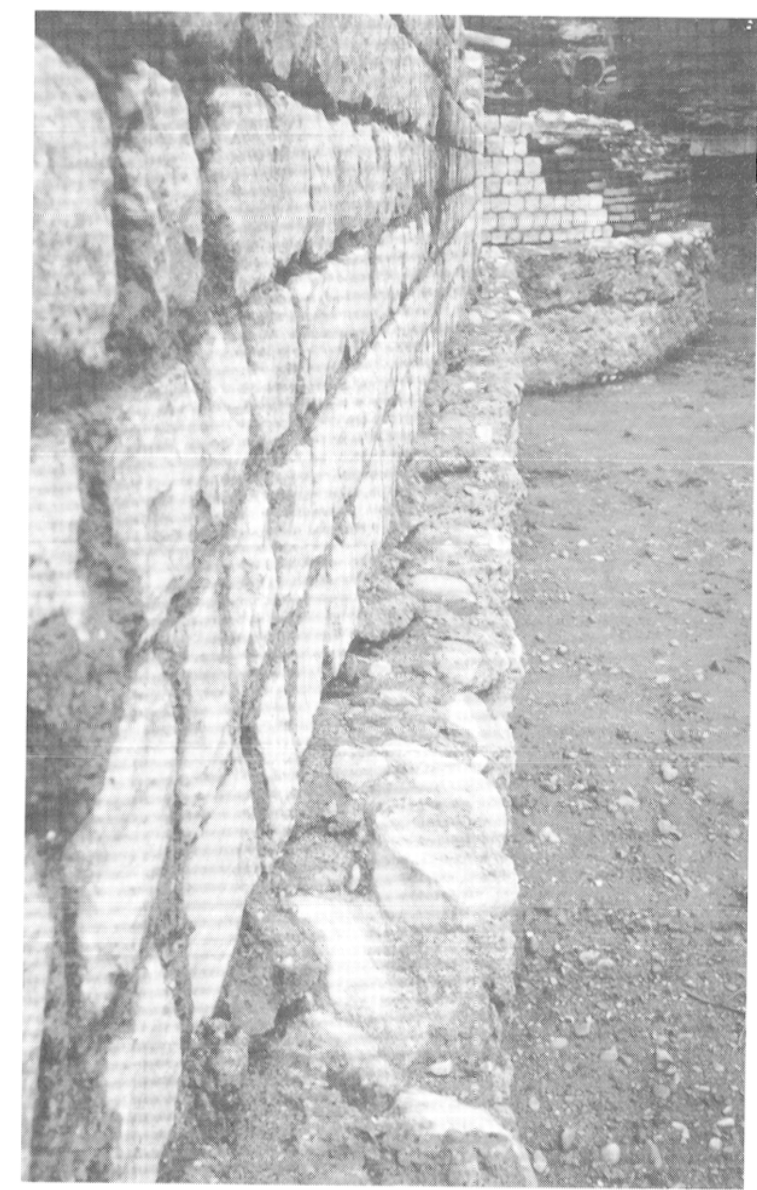

lig. 12 - La semelle de fondation de la courtine avec, en arrière plan, la nette distinction des deux niveaux superposés des banchées des fondations de la tour est.

une épaisseur constante au massif, répercute les mêmes variations : de 139,75 à $139,55 \mathrm{~m}$ (fig. 12).

\section{Le soubassement}

Le mur de l'enceinte, large de $2,40 \mathrm{~m}$, présente deux structures différentes entre la base et la partie supérieure de l'élévation. Haut de 1,40 m, le soubassement montre un parement en petit appareil coupé aux deux tiers par un chaînage de briques. Le petit appareil est constitué de moellons calcaires taillés en tronc de pyramide dont la queue, longue de 0,20 à $0,30 \mathrm{~m}$, pénètre dans le blocage en opus caementicium. Le matériau provient des calcaires à miliolites du Thanétien des Petites Pyrénées situées à quelque $70 \mathrm{~km}$ de Toulouse (Labrousse, 1968, p. 259). Les assises réglées ont, une hauteur de $0,11 \mathrm{~m}$. Des fondations au premier chaînage de briques, il faut compter normalement huit assises mises en valeur par des joints lissés au fer d'une épaisseur constante de $1 \mathrm{~cm}$.

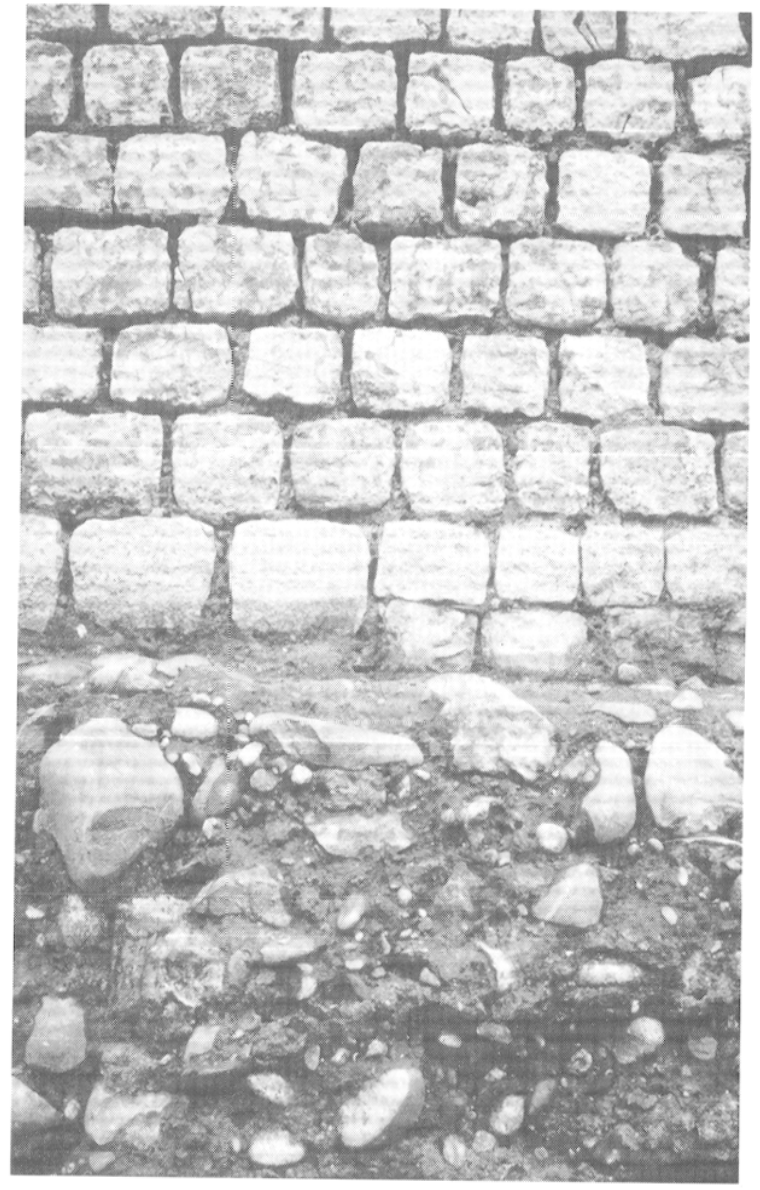

Fig. 13-Apport d'une assise supplémentaire de moellons à la base du soubassement.

Les variations du niveau de surface de la semelle de fondation qui s'aligne sur le relief du site sont corrigées par l'adjonction, à la base du soubassement, d'une assise supplémentaire. Celle-ci est amenée progressivement par des moellons de plus en plus gros, remplacés ensuite par deux rangs de petits mocllons dont la taille s'amplifie de nouveau sensiblement pour rattraper le module général (fig. 13). Le même procédé, inversé, permet de revenir au nombre initial d'assises lorsque la dépression est franchie. Le chaînage de briques est composé de trois assises qui traversent la totalité du mur pour apparaître sur les deux parements. Quatre autres assises de moellons calcaires surmontent le chaînage.

\section{L'élévation}

La liaison entre le soubassement et la partie supérieure du mur, montée intégralement en opus lestaceum, se fait par un second chaînage de trois 


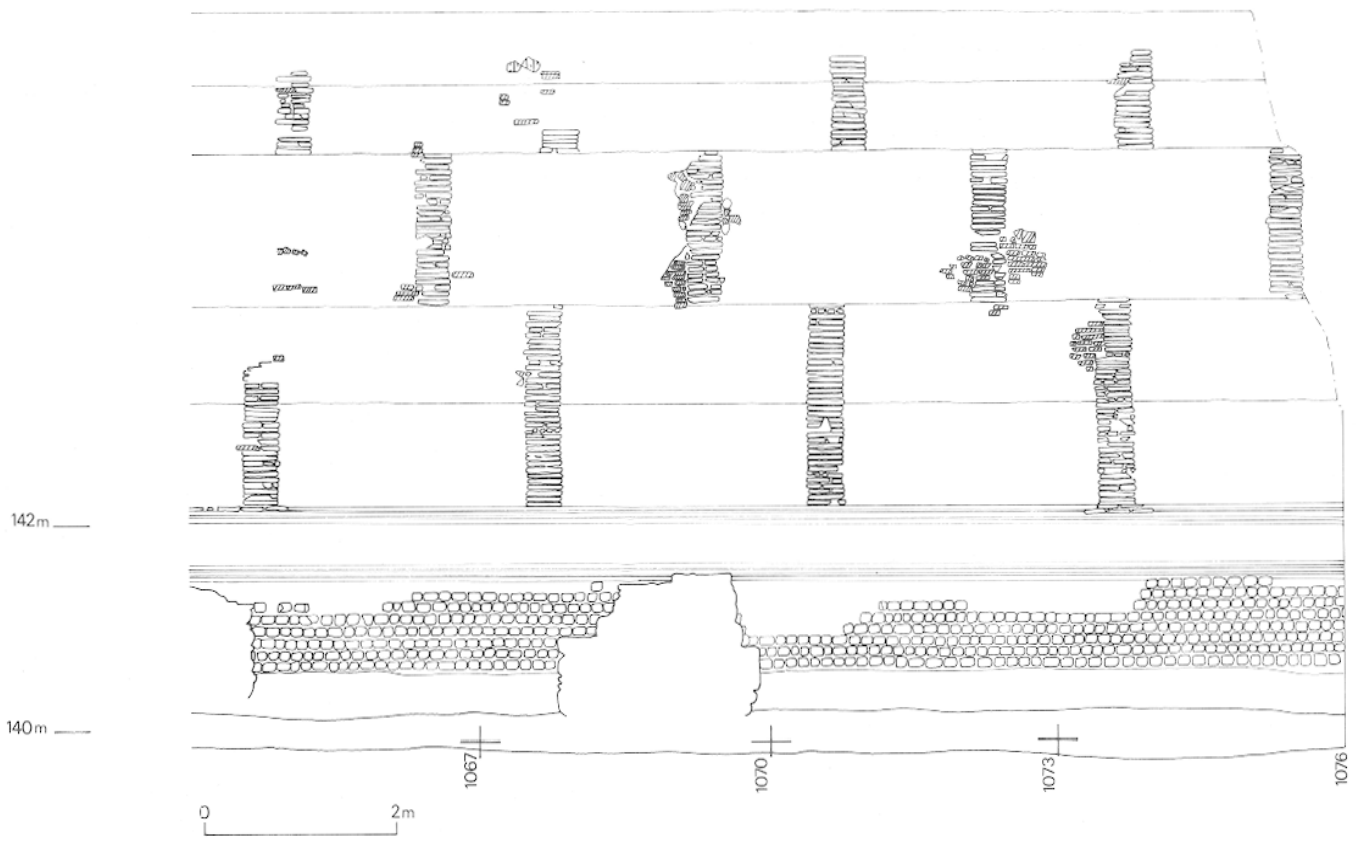

Fig. 14 - Relevé de la structure du blocage de la courtine dans sa partie est.

assises de briques qui assure ainsi le rôle technique de semelle de répartition. Les deux parements de briques, côté ville et côté campagne, identiques, ont une égale largeur de $0,60 \mathrm{~m}$. Leur somme équivaut à l'épaisseur du blocage qu'ils contiennent. Ce n'est pas seulement l'emploi exclusif de la brique en parement qui différencie la partie haute du mur, mais aussi la structure même du blocage. La spécificité du matériau a entraîné et permis cette technique. Le principe adopté pour monter le mur est celui du caisson de coffrage laissé en place pour constituer l'ossature de la structure définitive. I'utilisation des parements comme éléments même du coffrage est un procédé usuel de la maçonnerie romaine (Adam, 1984 , p. 79-80). Sur l'enceinte toulousaine, la technique est améliorée par l'adjonction, à intervalles réguliers, de murettes transversales qui relient les deux parements. Ces murettes de briques assurent le rôle de tirants qui soulagent les parements des poussées qu'ils subissent lors des opérations de banchage. En même temps elles compartimentent les banchées en volumes sans doute calculés selon les capacités de résistance des caissons de coffrage qu'elles constituent avec les parements (fig. 14).

Toutes les briques employées sur la courtine et les deux tours de l'enceinte à l'hôpital Larrey,

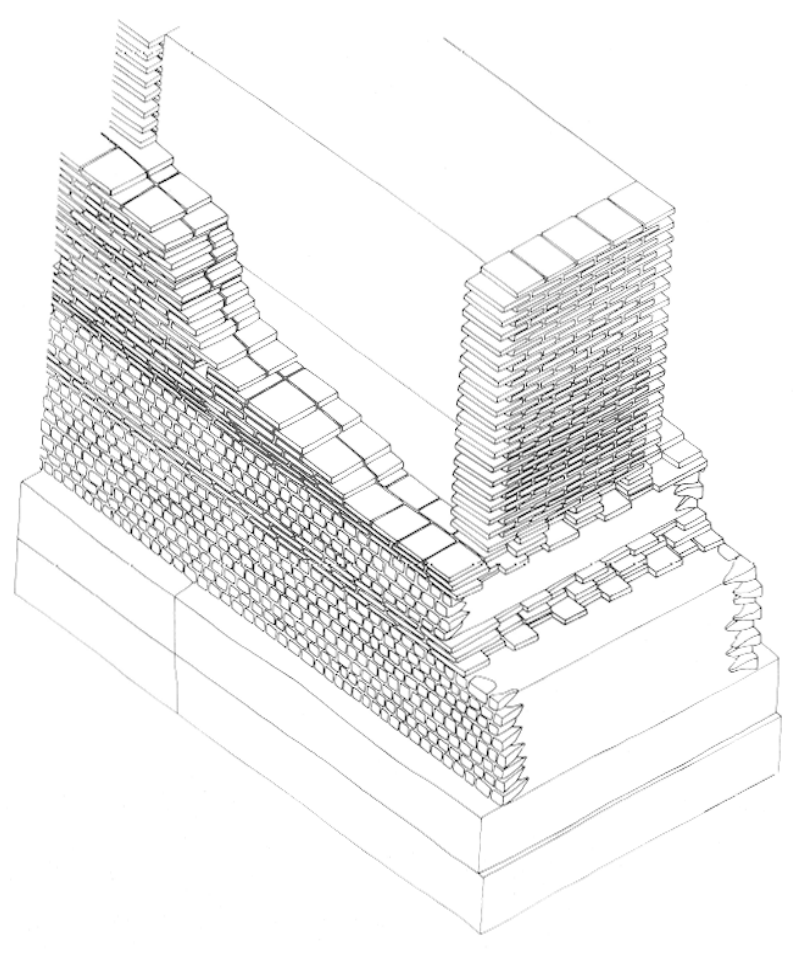

Fig. 15 - Schéma du système d'accrochage des murettes transversales du blocage avec les parements. 
qu'elles appartiennent aux chaînages horizontaux, aux parements ou aux murettes, ont été façonnées sur un seul module : $0,36 / 0,38 \times 0,22 / 0,24 \times 0,04 \mathrm{~m}$. Ce format a été retenu pour s'adapter parfaitement à l'unité de coordination dimensionnelle de l'ouvrage établie dès la conception : le pied romain. Ainsi, la largeur du parement équivaut à l'addition d'une longueur et d'une largeur de brique ( 2 pieds romains). Les briques du premier rang, celui qui parait, sont normalement disposées en boutisse et celles du deuxième rang en panneresse. Mais cet agencement n'est pas toujours possible, surtout pour l'aménagement des trous de boulin. La largeur d'une arase est égale à dix largeurs de brique $(2,40 \mathrm{~m}=8$ pieds romains). L'épaisseur du blocage et la longueur d'une murette comptent pour cinq largeurs. Toutes les assises des murettes sont composécs de la juxtaposition de cinq briques qui se côtoient par leur longueur. Chaque assise est décalée, par rapport à l'épaisseur du blocage, pour déborder d'une demibrique sur le parement. Ce décalage se fait alternativement assise par assise, de chaque côté du mur, et crée ainsi une saillie à redan qui permet d'effectuer la liaison entre les parements et les murettes transversales (fig. 15).

Les parements et les murettes de briques, distantes de 2,60 m, sont montés sur $1 \mathrm{~m}$ (18 assises) pour encaisser la première opération de banchage. Le premier plancher d'échafaudage est aménagé directement au-dessus du chaînage qui surmonte le soubassement, à 1,60 $\mathrm{m}$ du sol. Les boulins sont espacés de 2,40 m environ, s'intercalant ainsi aux murettes de briques. C'est un échafaudage encastré à un rang de perches. Les boulins ne pénètrent pas dans le blocage. Un trou de poteau repéré à $2 \mathrm{~m}$ devant le rempart, dans l'axe d'un trou de boulin, a dù caler une perche d'échafaudage. La cohésion de chaque cais- son, d'une capacité de $3,12 \mathrm{~m}^{3}$, permet aux parements de supporter le damage du garnissage où se mêlent en vrac mortier de chaux et galets de Garonne non calibrés.

Le deuxième niveau de banchage est pratiqué en surélevant d'une hauteur égale les mèmes murettes transversales (18 assises). Un plancher d'échafaudage est installé, comme le précédent, à la base du nouveau parement. La troisième opération de banchage se fait par l'implantation de nouvelles murettes disposées en décalage par rapport à celles du premier étage. Les nouveaux caissons sont montés, en une seule fois, sur une hauteur de 1,45 m (26 assises) et sont remplis par une banchée unique de $4,52 \mathrm{~m}^{3}$. Un plancher d'échafaudage a dù être aménagé au départ de ce niveau. Le troisième étage de caissons est toujours décalé par rapport à l'étage inférieur. Les murettes se rapprochent ainsi de l'axe de celles du premier étage. Ce dernier étage, élevé de $1,45 \mathrm{~m}$, est effectué en deux étapes égales $(2 \times 13$ assises) qui reçoivent chacune une banchée de $2,25 \mathrm{~m}^{3}$ (fig. 16). La disposition en damier imparfaite des murettes est calculée en fonction de l'emplacement des échafaudages.

La hauteur réelle du mur de l'enceinte toulousaine est inconnue. L'élévation de la courtine examinée à l'hôpital Larrey atteint $6,45 \mathrm{~m}$. Ce sommet correspond à l'arase d'une banchée et équivaut probablement au sommet réel de la structure à caissons de coffrage. La plus petite hauteur des banchées supérieures $(0,72 \mathrm{~m})$ est un argument favorable à cette hypothèse. Seul manquerait alors à la courtine décrite ici son couronnement, avec son chemin de ronde et son parapet à merlons et créneaux, ce dernier étant construit probablement totalement en briques.

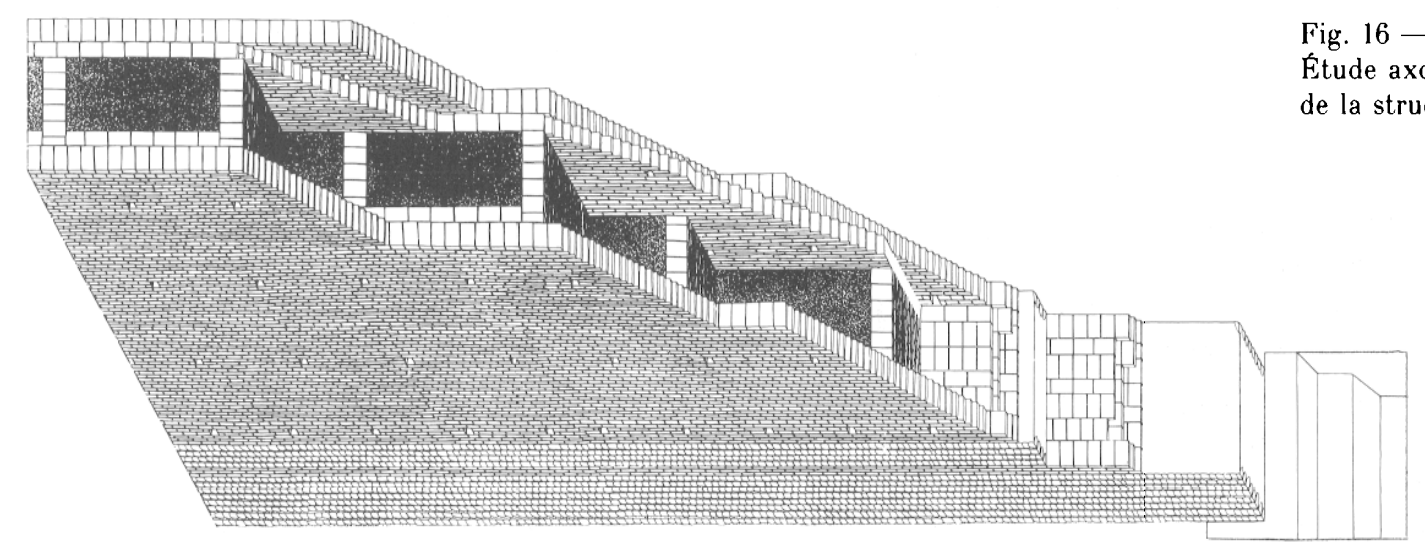




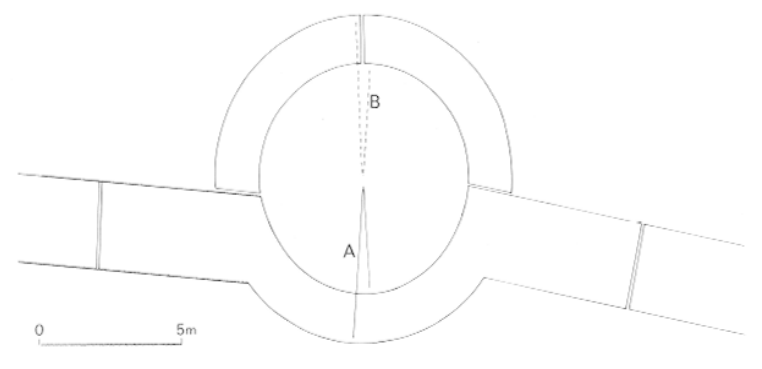

Fig. 17 - Schéma d'implantation des fondations de la tour est.

\section{Les tours}

Les deux tours, qui flanquent le mur d'enceinte et en interrompent le tracé, sont circulaires et creuses dès la base. La saillie de leur plan par rapport à la courtine, côté campagne, compte pour les trois cinquièmes de leur diamètre extérieur égal à $10,30 \mathrm{~m}$. Un examen minutieux de la circonférence des fondations de la tour est, aperçues dans leur intégralité, permet de reconstituer chacune des étapes de leur implantation. Les fondations de l'arc, côté ville, et les fondations attenantes des deux courtines y aboutissant ont été coulées en une seule longueur de banchée sur deux niveaux d'égale épaisseur, selon le principe général adopté. Les fondations de la partie frontale ont été réalisées dans un second temps. Le centre du cercle qui a servi à tracer leurs contours ne se superpose pas au centre du premier cercle utilisé pour la partie arrière de la tour (fig. 17A). Le décalage, peu conséquent, s'explique par l'implantation en deux temps de la circonférence de la tour. Entre la réalisation des deux étapes, le point de référence marquant le centre de la tour aurait été déplacé et son recalage n'aurait pas été correct (fig. 17B). Les fondations, en partie frontale, sont concrètement installées par moitié, en deux étapes successives (fig. 17).

Les tours, en élévation, reproduisent l'ordonnance des courtines : soubassement en petit appareil, avec chainnages de briques, et partie supérieure en opus testaceum. Le mème agencement ordonne le parement interne des tours. Les murs d'une tour apparaissent en épaisseur $(1,20 \mathrm{~m}$, avec un empattement des fondations de $0,20 \mathrm{~m}$ ) comme un dédoublement de la courtine, et leur structure, sans blocage interne, est uniquement en briques. La hauteur réelle des tours, supérieure à celle du mur de plusieurs mètres, et leur système de couverture restent eux aussi inconnus. Les reprises médiévales n'ont pas permis de repérer les seuils d'entrée.

La liaison entre la courtine et la tour ouest présente un agencement particulier. La construction de la courtine, longue de $51,35 \mathrm{~m}$ (entraxe des tours: $58,75 \mathrm{~m}$ ), a été entreprise à partir de la tour est. Seize murettes ou plutôt dix-sept caissons de coffrage ont été nécessaires pour contenir les banchées du premier étage de l'élévation en opus testaceum. Au départ, c'est le mur même de la tour est qui sert d'appui à la première banchée réalisée. A la jonction avec l'autre tour, l'intervalle entre celle-ci et la dernière murette compte $1 \mathrm{~m}$ supplémentaire. Cet écart est construit totalement en briques, masse interne comprise, et la maçonnerie est parfaitement liaisonnée à celle de la tour.

\section{A PROPOS DE L'ARCHITECTURE DE L'ENCEINTE}

La ville romaine est établie sur la terrasse alluviale qui domine d'une quinzaine de mètres la rive droite de la Garonne, sur la côte convexe d'un méandre du fleuve, dans un secteur non inondable (Labrousse, 1968, fig. 1). Le tracé général de l'enceinte, long de $3 \mathrm{~km}$, décrit une vaste boucle ouverte sur le fleuve d'une surface de 90 ha. Sur le terrain l'implantation de l'ouvrage s'adapte à la topographie du site : elle suit de légères lignes de crête et évite les zones marécageuses (Baccrabère, 1977, p. 16-20). Le front fluvial sera barré par l'adjonction d'une muraille complémentaire au Bas-Empire (Baccrabère, 1974).

Le plan de Tolosa le plus récent, dessiné par les services d'urbanisme de la Ville de Toulouse à l'occasion d'une exposition consacrée à la cité romaine (Palladia Tolosa, 1988), présente une configuration de l'enceinte fondée sur des données précises, soulignant les parties observées et relevées réellement, et distinguant les éléments restitués et les éléments seulement connus par des documents anciens (fig. 1). Le parcours de la muraille est ponctué d'une cinquantaine de tours qui partagent les courtines en longueurs assez régulières, autour d'une moyenne de $45 \mathrm{~m}$. Des deux portes principales, placées dans l'axe du cardo maximus, seule l'ordonnance de "la Porterie", au nord de la ville, est connue (Labrousse, 1971). Les tours sont bâties sur deux modèles. Les tours circulaires, les plus nombreuses, sont toutes identiques. Les tours à talon carré, de plus grandes dimensions, ont leur avant-corps semi-circulaire ou polygonal. L'agencement préconisé par l'abbé Bac- 
crabère (1977, p. 43) qui dispose régulièrement une tour à talon après trois tours rondes est confirmé par les découvertes les plus récentes. Finalement, si dans la ville actuelle, les vestiges de l'enceinte sont peu visibles, peu de tronçons restent encore aujourd'hui totalement inconnus. Hormis le secteur nord-est, les éléments explorés se répartissent d'un bout à l'autre du tracé, où une majorité de tours ont été aperçues intégralement ou en partie.

De la totalité des observations émises par l'ensemble des inventeurs de l'enceinte toulousaine, il en est une, essentielle : l'édifice dans son développement, son agencement, son architecture et ses techniques de construction, est uniforme. Cette régularité probante implique l'unicité de conception et de réalisation de l'ouvrage dans des délais brefs. Cette assertion autorise l'application des données acquises sur le site de l'hôpital Larrey à l'ensemble de l'enceinte de Toulouse, et cela autant pour la description du monument que pour la détermination de sa datation.

La variation du nombre d'assises du soubassement est due à des incidences topographiques (rattrapage de niveau); le deuxième chaînage de briques, qui fait la liaison entre le soubassement en petit appareil et l'élévation en opus teslaceum avec laquelle il se confond en parement, n'avait jamais été relevé. Si l'analyse architecturale des vestiges dégagés à l'hôpital Larrey amène des précisions quant à l'ordonnance externe de l'enceinte, c'est surtout sur la connaissance de sa structure interne et de son mode de construction que les progrès sont remarquables. La preuve que la structure des tours, en élévation, est bâtie intégralement en briques, doit amener à reconsidérer l'origine des parties supérieures des tours encore visibles dans la ville actuelle (Labrousse, 1968, p. 237) et dont l'antiquité est encore contestée malgré les allégations de A. Soutou (1969). L'observation inédite de la façon d'installer les fondations en deux niveaux d'égale épaisseur et la reconnaissance des longueurs de banchées permettent de calculer la capacité d'une seule opération de banchage nécessitant, pour le cas le mieux attesté, plus de $19,53 \mathrm{~m}^{3}$ d'opus caementicium. Le repérage des raccordements des diverses banchées dont le front est parfois nettement incliné donne également le sens de progression des travaux. L'exploitation de ces informations nouvelles devrait apporter des indices sur la durée même du chantier de construction et concerne donc la question de la datation globale de l'enceinte. Cette démarche est à poursuivre sur les diverses parties de l'élévation où, là aussi, toutes les opérations de banchage ont été dis- tinguées. Le compte des matériaux préfabriqués (moellons calcaires et briques) et le calcul de la masse du blocage (galets et mortier de chaux) sont envisageables. L'avancement des travaux sur l'enceinte dépend grandement des cadences de production et d'approvisionnement des matériaux. Ce dernier corollaire n'est pas le moindre des paramètres qui interviennent dans le fonctionnement d'un chantier de cette envergure et qui assurent à l'ouvrage achevé son uniformité : c'est le caractère fondamental de la première enceinte de Toulouse.

\section{Les innovations de la maçonnerie romaine}

Petit appareil, arases de briques, opus testaceum, l'enceinte de Toulouse profite des plus récentes innovations de la construclion romaine, à une époque où, à Rome, elles n'apparaissent encore que timidement dans l'architecture publique. Les nouvelles formules trouvent un plus large champ d'application là où les schémas urbanistes gréco-romains sont eux-mêmes les promoteurs de la romanisation. Ce sont les enceintes augustéennes des villes de fondation récente ou dont le statut juridique évolue, notamment en Cisalpine et en Narbonnaise, qui bénéficient les premières de l'emploi généralisé du petit appareil (Adam, 1984, p. 148). Toulouse ne possèdant pas de carrières de pierre dans son environnement immédiat se procure ce matériau à plus de $70 \mathrm{~km}$. Dans l'enceinte, son usage est restreint au soubassement nécessaire au mur de briques, non pour encaisser les chocs du bélier. mais pour protéger l'ouvrage du dégravoiement. La première arase de briques qui partage le petit appareil se trouve au sommet de la première banchée du soubassement pour régulariser un plan horizontal corrigé sur le registre inférieur par l'apport d'assises complémentaires. La deuxième arase établit la transition avec la superstructure en briques. La présence précoce de ces assises de réglage dans la maçonnerie romaine peut valider l'observation faite jadis de cette technique sur l'enceinte de Vienne (Le Bot-Helly, 1987, p. 52). Elle est aussi clairement attestée sur les courtines de l'enceinte de Turin à parement externe de briques (Lugli, 1957, p. 623). Turin et Toulouse, deux villes portuaires fluviales, doivent leur fortune à leur situation en des points de rupture de charge (Mansuelli, 1971, p. 59; Labrousse, 1968, p. 102) et leur architecture de briques procède d'une même carence locale de matériaux de pierre. C'est aussi le fleuve, par l'exploitation de ses graves, qui fournit le garnissage de la structura caementicia de leur muraille. Les enceintes de Vérone, sur l'Adige, et de Turin, dont les portes 
majestueuses sont préservées, sont les premières réalisations monumentales en opus testaceum dont l'émergence a été tributaire de celle de l'opus caementicium. L'expansion de l'appareil de briques dans l'architecture romaine coïncide avec les débuts de l'Empire (Mansuelli, 1971, p. 112-113). Il est utilisé à Toulouse sur tous les grands monuments connus de la ville antique, datés du $\mathrm{I}^{\mathrm{er}} \mathrm{s}$. : enceinte, amphithéâtre, théâtre (Palladia Tolosa, 1988, notamment Rico, 1988, p. 84-87). La construction intégrale en briques d'un fanum de type celtique, au milieu ou dans la seconde moitié du $\mathbf{I}^{\mathrm{er}} \mathrm{s}$. avant J.-C., sur l'emporium de Vieille-Toulouse (Vidal, 1988, p. 12) est le témoignage le plus précoce de l'intrusion de ce nouveau matériau dans une architecture indigène. $\Lambda$ Toulouse, lors d'une récente opération archéologique associée aux travaux du métro, a été découvert, sur le parcours du cardo maximus, intégré au portique du forum, un édicule doté d'un parement en véritable opus mixtum sur lequel alternent régulièrement deux assises de briques pour une assise de moellons calcaires (de Filippo, renseignement inédit). La datation aux deux premières décennies de notre ère de cette structure prouve également la précocité de la mise en wuvre des divers modes de construction liés à la brique dans la cité des Tolosales. Leur apparition est jugée plus tardive dans l'ensemble des Gaules (Adam, 1984, p. 154). G. Lugli s'étonnait déjà du faste de l'architecture de la Porte Palatine de Turin et de l'usage aussi parfait de la brique quand à Rome ou ailleurs elle est encore ignorée (Lugli, 1957, p. 575). Si le programme de ses portes paraît avoir été plus modeste que ceux des portes de Vérone et de Turin, c'est la haute technicité appliquée à la construction de ses murs qui surprend le plus sur l'enceinte de Toulouse. Leur structure à caissons n'a pas, semble-t-il, d'équivalent. Son élaboration satisfait aux exigences du maçonnage du gros ouvre : la solidité des coffrages permel à l'ouvrage de résister aux opérations de damage; le compartimentage correspond aux normes admises pour d'autres techniques (Adam, 1984, p. 82, 83). Elle résoud également, grâce à son ossature, les problèmes architectoniques: les raidisseurs maintiennent les parements, contiennent les poussées et absorbent les chocs. I a répartition alternée des murettes par étage accroît l'efficacité du procédé dont la systématisation induit, et ce n'est pas le moindre de ses avantages, la rationalisation du travail. I'organisation du chantier est définie par l'assignation hiérarchisée des tâches, simplifiées et répétitives, rendue possible par la commodilé des matériaux mis en cuvre: la brique et l'opus caementicium.
Les ingénieurs romains emploient le système des boutisses pénétrant dans le massif en opus caementicium pour assurer une meilleure solidarité entre les parements à grand appareil rectangulaire à assises réglées et le blocage interne (Adam, 1984, p. 236). Les exemples les plus évocateurs de son application appartiennent aux structures massives de quelques monuments funéraires de la via Appia en opus quadralum (I.ugli, 1957, pl. CVI). Mais à notre connaissance, aucune structure à caissons semblable à celle des murs de Toulouse n'est signalée ailleurs que dans cette même ville, sur le Rempart de Garonne édifiè à partir de la fin du $\operatorname{II}^{\mathrm{e}} \mathrm{s}$. pour protéger le front fluvial (Baccrabère, 1974). Il exisle peu de remparts en opus lestaceum et ceux de Vérone el de Turin, de la seconde moitié du $\mathrm{I}^{\mathrm{er}} \mathrm{s}$. avant J.-C. (Rebechi, 1987 , p. 140, 145), sont aussi les premières grandes réalisalions en briques de la maçonnerie romaine. L'ouvrage toulousain, peut-être "la dernière des enceintes augustéennes" érigées en Narbonnaise, techniquement très élaboré, profite-t-il, grâce à l'expérience acquise, des progrès de la mise en ceuvre du nouveau matériau? Est-ce le décalage de datation avec les rares précédents cisalpins qui explique la spécificité de son mode de construction, mis au point entretemps?

C'est à partir de l'apparition de la maçonnerie concrète, à la fin du $1 \mathrm{II}^{\mathrm{e}} \mathrm{s}$. avant J.-C., que l'architecture romaine se démarque de l'influence grecque en élaborant de nouvelles formules. La mise en cuvre de l'opus caementicium entraîne l'émergence de nouveaux types d'appareil tels que l'opus incertum et l'opus reliculalum. Cette évolution est flagrante dans la construction des enceintes à l'origine en appareil mégalithique et dès le $\mathrm{II}^{\mathrm{e}} \mathrm{s}$. en maçonnerie concrète. Elle est encore perceptible sur la muraille de Terracina, en pays volsque, qui a conservé le témoignage de ces étapes successives (Adam, 1984, p. 113). L'opus incertum se retrouve sur les nombreuses fortifications élevées en Italie au moment des Guerres civiles (Adam, 1984, p. 139). L'opus quasi-reticulatum et l'opus reticulalum sont employés sur les murailles d'Ascoli, dans les Marches, et de Saepinum, dans les Abruzzes, dans le couranl, du $\mathrm{I}^{\mathrm{er}} \mathrm{s}$. avant J.-C. et au changement d'ère (Rebecchi, 1987, p. 135, 142). L'essor du petit appareil est étroitement lié à la construction des enceintes augustéennes. Il a déjà été démontré que les premières grandes réalisations en opus testaceum concernent aussi ces ouvrages. Comme si ces vastes programmes, moins contraints par la tradition ou les règles attachées, par exemple, aux édifices cultuels, avaient offert un meilleur champ d'application aux nouvclles formules. Surtout, ces 
techniques sont une réponse aux mutations socioéconomiques du monde romain (Adam, 1984, p. 144) : elles permettent la systématisation du travail, par une standardisation des matériaux et des tâches, approprièe, en exceptant les portes, à "l'envergure monotone" de ces chantiers.

\section{Les rémanences de la poliorcétique hellénistique}

Les règles de l'architecture antique spécifiques aux enceintes préconisent l'emploi de chaînages dans leur construction. Ces assemblages sont prescrits par Philon de Byzance dans l'édification des fortifications; ils sont présents sur les murs d'Athènes où ils sont en bois (Martin, 1965, p. 4-5). Si les chaînages structurent le travail des bâtisseurs, le théoricien de la poliorcétique insiste sur leur rôle premier: ils constituent l'armature indispensable à un ouvrage défensif prêt à supporter les assauts (I, 12). Vitruve recommande aussi cette ossature $(I, 8)$ et César la découvre dans le murus gallicus (Guerre des Gaules, VII, 23). A regarder le segment de murus gallicus reconstitué à Vertault, en Côte-d'Or (Goudineau, 1990 , p. 297), comment ne pas y voir une évidente analogie de structure avec le rempart de Toulouse? Le procédé appliqué à ce dernier correspond à une règle universelle $\mathrm{du}$ génie militaire adoptée par diverses communautés culturelles. Son élaboration apparait alors sous des formules différentes adaptées aux traditions architecturales locales. La particularité de l'enceinte toulousaine réside dans cette adaptation d'un nouveau matériau à une formule dont l'origine se situe dans le monde grec et dont la conception est spécifique à la construction des enceintes.

$\mathrm{Au} \quad \mathrm{IV}^{\mathrm{e}}$ s., apparaissent dans l'architecture grecque les premiers remparts conçus avec deux parements contenant un remplissage de terre et de pierraille, selon la technique de l'emplecton décrite par Vitruve (II, 8). Ils succèdent aux murs à corps massif en pierre de taille ou en briques crues, dispendieux en coût et en délais de réalisation (Adam, 1981, p. 15). Les ouvrages en briques crues, préférés pour leur plus grande résistance aux chocs, reposent généralement sur un socle de pierres qui les protège du ravinement (Martin, 1965, p. 358). L'inconvénient majeur des murs à double parement, toujours en appareil rectangulaire, est leur manque d'homogénéité. Pour pallier ce défaut, des boutisses pénètrent dans le remplissage. L'aménagement de murets transversaux qui relient carrément les parements et compartimentent le garnissage de tout-venant per- fectionne la cohésion et la solidité de la structure (Garlan, 1974, p. 199). Sont ainsi faites les murailles du cap Sounion en Attique et de Géla en Sicile (Adam, 1981, p. 34, photo 52; p. 35, photo 57). En Italie, les murs grecs de Naples sont bâtis selon la même technique, adoptée également par les Étrusques dans l'enceinte de Bolsena notamment (Lugli, 1957, p. 299-300, fig. 78 et p. 278, fig. 72, pl. LVIII, 2).

Dans les murs grecs et étrusques à grand appareil rectangulaire, les parements sont les éléments porteurs. Leur masse interne n'est pas un blocage, mais un simple bourrage de matériaux divers non cimentés. Dans les murs romains, en maçonnerie concrète, la règle est inverse : le blocage en opus caementicium est le massif porteur; les parements en petit appareil ne sont qu'un "habillage». La façon de dresser les murs est aussi radicalement différente. L'espace réservé au garnissage est rempli après exécution des parements à grand appareil qui supportent les opérations de damage; l'emploi de boutisses ou de murets transversaux permet la cohésion de l'ensemble et, pour les remparts, donne une armature indispensable. Dans la construction romaine, seuls les grands édifices en opus quadratum (les mausolées de la via Appia, avec boutisses) ou en opus testaceum (l'enceinte toulousaine, à murets transversaux) autorisent cette technique, conjointement à la maçonnerie concrète (Adam, 1984, p. 82). Ce procédé est incompatible avec le petit appareil, qu'il soit réticulé ou réglé. Même les arases y sont rarement incorporées à l'origine. Elles apparaissent tardivement dans les enceintes, à Vérone et à Turin, sous la forme de deux assises de briques, simultanément à l'opus testaceum (Lugli. 1957, p. 518); ensuite peut-être à Vienne et dans le soubassement en petit appareil à Toulouse. Là et plus fréquemment dans les enceintes gallo-romaines $\mathrm{du}$ Bas-Empire, intercalées entre deux opérations de banchage, les arases sont moins des chaînages que des assises de réglage qui ajustent le travail des bâtisseurs.

Si l'usage de la maçonnerie concrète modifie totalement les règles de l'architectonique classique dans la construction romaine, celle-ci reste malgré tout tributaire des schémas grecs (Gros, 1978, p. 6). Il en est ainsi, comme le démontre F. Rebecchi (1987, p. 133-135), des lois de la poliorcétique hellénistique dans la conception des enceintes tardo-républicaines et augustéennes. Cette rémanence transparaît particulièrement dans les portes à tenaille de Fréjus, d'Arles et d'Aix-en-Provence, ou encore dans la porte "scéenne» de Vintimillc : les dcux modèles sc trouvent dans la même muraille de Mantinée en 


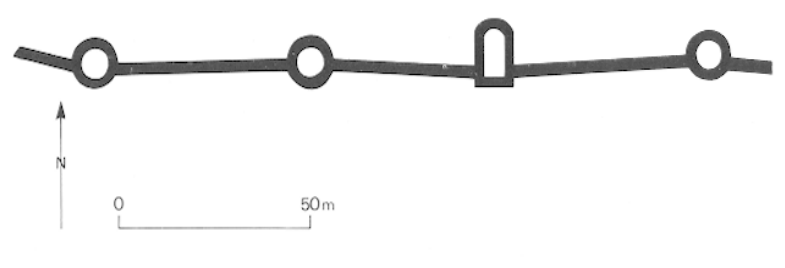

Fig. 18 - Tracé à décrochements de l'enceinte dans sa partie terminale nord-ouest (du lycée Pierre de Fermat à la place Saint-Pierre).

Arcadie édifiée au $I^{*} \mathrm{~s}$. Parmi les préceptes diffusés par les théoriciens grecs et adoptés par les ingénieurs romains, certains caractérisent de façon essentielle l'enceinte de Toulouse. Les murs d'une ville ne sont efficaces que s'ils pourvoient à leur propre protection par des ouvrages de flanquement qui segmentent les courtines en longueurs pouvant être couvertes par le champ de tir des armes de jet. La distribution et donc le nombre de tours de l'enceinte toulousaine dépendent directement de l'application stricte de cette règle. La plupart des courtines mesurent $45 \mathrm{~m}$ ou environnent cette valeur qui correspond à peu près à 150 pieds romains. Peu sont inférieures à $40 \mathrm{~m}$ ou supérieures à $50 \mathrm{~m}$. Le flanquement est davanlage perfectionné par le tracé à décrochements des courtines dont le système, vérifié sur le site de l'hôpital Larrey, s'inspire des crémaillères bastionnées de la poliorcétique hellénistique (Garlan, 1974, p. 248), (fig. 18). Le plan même des tours, circulaire ou à talon carré et front curviligne ou polygonal, améliore encore le champ de surveillance et accroît leur capacité de résistance (Adam, 1981, p. 57, 62). La technique de construction de ces ouvrages suit aussi les principes recommandés : leurs murs, deux fois moins épais que ceux des courtines, sont appareillés dans toute leur largeur (Adam, 1981, p. 32). L'implantation compartimentée des fondations d'une tour, où la partie frontale la plus menacée est exécutée dans un deuxième temps, permet peut-être d'assurer, en cas de dommages, l'intégralité du reste de l'ouvrage solidaire des courtines attenantes. Enfin, l'unique plan connu d'une porte, avec son cavaedium circulaire non ouvert sur les passages piétonniers latéraux, résulte de la prise en compte de toutes les précautions relatives à la défense des accès d'une ville ceinturée.

La conception de l'enceinte de Toulouse témoigne de cette acculturation significative de l'architecture romaine dans le domaine militaire. F. Rebecchi observe les manifestations de ce processus en Italie au dernier siècle de la République et au début du Principat (1987, p. 133-135). Cette connexion des préceptes rémanents hellénistiques et des techniques novatrices de la construction romaine intervient même à Toulouse dans le choix du matériau. Est-il encore possible, après la correction de son anachronisme, d'affirmer que seul le défaut endémique de pierre a déterminé l'utilisation de la brique, de toute façon non exclusive, sur cette enceinte? Après tout, l'unique argument dirimant contre une datation haute consistait en la seule présence d'un chaînage de briques dans le soubassement en petit appareil, si concluant pour exclure totalement de la discussion l'opus teslaceum de son élévation. Il n'est pas fortuit que les premières grandes réalisations en briques de l'architecture romaine soient spécifiquement des enceintes. L'exemple turinois est flagrant: seule la face exposée, côté campagne, est pourvue d'un appareil de briques. Et à Rome, le Castro Prelorio est bien le premier édifice bâti ainsi, sensiblement à la même époque que l'enceinte toulousaine.

\section{A PROPOS DE LA DATE DE L'ENCEINTE}

La date de l'enceinte induite des résultats avérés obtenus sur le site de l'hôpital Larrey incite immanquablement à poser une nouvelle fois la question de la date de l'accession de la cité de Toulouse au statut de colonie. Cette incidence entre «enceinte et colonie", à peine esquissée par M. Labrousse (1968, p. 276), postulée par P.-A. Février (1969), est renouvelée depuis une récente proposition de J.-M. Pailler : l'empereur Domitien élève la cité au rang de colonie romaine dont l'insigne ostensible est l'enceinte du chef-lieu (Pailler, 1988). Si cette dernière et séduisante inférence ne peut plus être soutenue à la lumière des données archéologiques acquises présentement, l'axiome reste cependant "à discuter" dans le nouveau cadre chronologique.

A l'hôpital Larrey, la courtine construite vers 30 après $J$.-C. est située au nord-ouest de la ville, où le parcours de l'enceinte aboutit bientôt sur la rive de la Garonne. Là, l'examen des fondations établit que les travaux progressèrent en direction du fleuve. Cette indication majeure autorise à présenter le déroulement probable du chantier : les portes, érigées les premières, furent les points de départ des diverses phases, sûrement concomitantes, de réalisation des courtines. Ainsi, il n'est pas possible de restituer l'exécution de la tranche ouest, de "la Porterie" à la Garonne, dans l'évolution globale du projet. Il est aussi hasardeux de placer les indices de datation au terme de cette même opération en raison de la 
gradation différenciée des postes de travail. Une seule proposition reste alors acceptable : l'enceinte toulousaine est en cours de réalisation en 30 après J.-C. La durée estimée de cette construction ne doit pas excéder la décennie. Cette appréciation s'appuie sur les études relatives à la muraille de Saepinum, dans les Abruzzes (Coarelli, La Regina, 1984, p. 215) et sur l'évaluation indiquée pour l'enceinte de Nîmes (Gros, 1991, p. 44). Le développement moindre de son tracé et son mode de construction particulier impliquent un délai d'exécution plus bref pour l'enceinte de Toulouse. Le démarrage des travaux est précédé d'une phase d'élaboration dont l'estimation est plus conjecturale : prévisions financières, définition architecturale (tracé, plan, construction), projection de besoins (matériaux, main d'œuvre). La déduction de ce processus, à rebours d'une datation relative à sa phase de matérialisation, conduit à cerner davantage le contexte initial auquel le projet est subordonné.

La plupart des vastes enceintes gallo-romaines du Haut-Empire, reconnues, sont érigées à l'époque augustéenne en Narbonnaise, où elles appartiennent à des cités dotées du statut de colonie romaine ou latine (Goudineau, 1980, p. 244, fig. 166). Hors de cette province, seule l'enceinte d'Autun est contemporaine et comparable. Si son statut privilégié de "fédérée" ne peut expliquer seul cette particularité, il n'est pas improbable que la cité des Éduens soit nantie, dès cette époque, du titre de colonie (Gros, 1987, p. 162). L'existence d'une enceinte à Lyon est problématique; celles de Nyon et d'Augst, en territoire helvète, sont rudimentaires. Quatre enceintes sont plus tardives : à Cologne en Germanie Inférieure, à Avenches en Germanie Supérieure, à Tongres et à Trèves en Gaule Belgique. Ce tableau chronologique, généralement admis, ne s'appuie pas toujours sur des datations pleinement assurées. Des confirmations ou des corrections pourront résulter d'éventuelles découvertes archéologiques. Ainsi, récemment, une opération de sauvetage a permis de reporter la datation de l'enceinte de Fréjus à la fin du $\mathrm{I}^{\mathrm{er}}$ s. (Pasqualini, 1992, p. 29, n. 111).

Les enceintes augustéennes de Narbonnaise ne se définissent pas uniquement dans un contexte spatio-temporel homogène et par des caractéristiques structurelles analogues. Elles participent d'un processus de plus grande ampleur dont elles affermissent la cohérence. A la suite de la Guerre sociale, l'accession des Alliés à la citoyenneté inaugure en Italie un vaste programme d'urbanisation dans lequel la construction d'enceintes apparaît comme le signe ostensible de cette émancipation. Dès lors, dans la péninsule et cela jusqu'à l'avènement d'Auguste, à la suite d'une fondation coloniale ou d'une élévation juridique, l'enceinte "se fait voir comme l'expression excentrique" de la rénovation urbaniste et monumentale qui accompagne la promotion d'une cité. Vérone demeure le meilleur exemple de ce processus en Transpadane au moment de la Lex Iulia et les cités de Narbonnaise de son extension dans les provinces occidentales à l'époque augustéenne (Rebecchi, 1987; Gros, 1987, p. 160). Cependant cette déviation sémiologique de l'enceinte n'estompe aucunement sa fonction défensive. Les règles de la poliorcétique sont respectées. Souvent l'ornementation ostentatoire d'une porte monumentale revêt un ouvrage de conception strictement militaire (Rebecchi, 1987, p. 134-135).

L'ambivalence conceptuelle de l'enceintè précise davantage l'ambiguité des fondations coloniales en milieu provincial à la fin de l'ère républicaine et au début du Principat. En Gaule, ces pôles de la romanité sont aussi, durant cette période, les vigies de la pacification (Goudineau, 1991, p. 10-12). La répartition des colonies de vétérans établies par César ou Octave dans le couloir rhodanien et en pays helvète semble répondre à des motifs stratégiques complexes. La centuriation des terroirs et l'urbanisation des chefs-lieux sont les manifestations de cette romanisation propagandiste et civilisatrice. Là, l'enceinte de la cité, annonciatrice de la Pax Romana (sécurité et prospérité), est l'expression tangible de cette définition duale de la romanité installée. Toutefois, il est des villes dont l'enceinte affirme davantage l'objectif de la fondation coloniale. Les remparts de Nyon et d'Augst, palissades de bois et levées de terre, avec fossés, semblables aux premières fortifications de Tongres et sans doute à celles de Narbonne à ses origines (Gros, 1987, p. 161), tiennent sans conteste des positions militaires. A l'opposé, les enceintes des colonies de droit latin (Aix, Nîmes) instituées par Auguste accompagnent moins une promotion purement honoraire que la faveur d'un patronage impérial consentie avec parcimonie à des cités qui entretiennent des relations déjà anciennes avec Rome (Le Gall, Le Glay, 1987, p. 125). Accordées, voire offertes par le Princeps (CIL XII, 3151), elles déclarent aussi l'accomplissement d'une mutation urbaniste qu'elles cadrent, à l'instar du péribole du forum dont elles répercutent le symbolisme... Après ces enceintes augustéennes de Narbonnaise, auxquelles il faut joindre celle d'Autun, les constructions de cet ordre sont rares. Exception faite désormais de l'enceinte de Fréjus, elles sont toutes situées sur le limes germanique. Là, leur «décalage chrono- 
logique» se comprend par la position géostratégique sensible des villes qui participent à la consolidation d'une zone déjà fortement militarisée. Leur caractère de forteresse est plus accentué. Ainsi, par sa rigueur, l'ouvrage de Cologne rappelle celui d'Aoste qui fut créé pour être un des bastions de la pacification des Alpes (Rebecchi, 1987, p. 146). Par ailleurs, les cités qui obtiennent, après Auguste, le titre de colonie ne construisent pas d'enceinte (Goudineau, 1980, p. 315 , fig. 241).

Les découvertes les plus récentes de l'archéologie toulousaine, amenées opportunément grâce aux travaux du métro, démontrent que le cardo maximus (et sans doute l'ensemble du réseau, avec l'équipement des égouts) et le forum de la ville romaine sont implantés lors d'un même chantier ouvert dans le courant de la deuxième décennie de notre ère. Ces réalisations, concomitantes, procèdent d'un vaste et unique programme d'aménagement dans lequel s'inscrit sûrement, eu égard à la chronologie, la construction de l'enceinte. Cette dernière, conçue sous Tibère, fut peut-être déjà prévue par Auguste. Ici, la discussion ne doit pas seulement établir que la cité des Tectosages acquiert le titre de colonie préalablement à ses murs. Pour les Gaules, le constat est évident: seules les colonies, romaines ou latines, obtiennent ce privilège (Gros, 1987, p. 162). Inventoriée par Pline l'Ancien parmi les oppida latina $(\mathrm{NH}$, III, 37), qualifiée de colonie romaine par l'tolémée (Géographie, II, 10, 6), Toulouse la Palladienne, à défaut d'une épigraphie lacunaire, n'a d'autre référence que son enceinte admirable pour réhabiliter sa qualité de "cité augustéenne» qu'elle doit à l'intervention directe du Princeps ou à celle de son coadjuteur el successeur Tibère.

\section{LES ORIENTATIONS DE RECHERCHE}

L'enceinte de Toulouse semble surprenante dans la mesure où, en Gaule romaine, elle demeure l'unique exemple d'une conception architectonique fondée essentiellement sur l'emploi de la brique. Elle apparaît si aberrante qu'elle est exclue, comme les autres monuments toulousains tous revêtus de briques, des études relatives à la construction galloromaine. Cette marginalisation vaut même à Toulouse une espèce de damnatio memoriae qui la condamne encore aujourd'hui à ne point être considérée comme "une ville augustéenne».

A Toulouse, la brique passe pour un matériau de substitution tellement normal qu'il n'a jamais vrai- ment été posé de question à ce sujet. Seuls les chaînages du soubassement de l'enceinte, parce que le système est connu ailleurs, ont été utilisés pour la datation de ce monument. Le fanum de Vieille-Toulouse, l'amphithéâtre de Purpan, le théâtre de la ville, jusqu'à présent considérés comme antérieurs, n'ont rien apporté à l'affaire. Pourtant, et le paradoxe est bien là, la nature du programme architectural, l'ampleur du projet, les rémanences hellénistiques auraient dû suffire à placer cette enceinte parmi ses analogues de Narbonnaise.

A l'instar de Nîmes et de Vienne, Toulouse est bien le chef-lieu d'une cité de droit latin qui mérite son titre de colonie accordé peut-être par $\Lambda$ uguste lui-même. Les enceintes de ces trois villes, plus encore que celles des colonies romaines qui les possèdent de droit, sont "données" par et pour le Princeps, elles marquent la célébration légitime de sa protection personnelle. Leur conception montre qu'elles sont plus qu'une parure monumentale régalienne. La tradition romaine prévaut toujours lorsqu'il s'agit d'édifier un rempart: la fonction militaire est prioritaire. Ainsi, les ouvrages à tours carrées des villes d'Aoste et de Cologne ont été conçus selon les règles de la castramentio. $\Lambda$ l'opposé, les enceintes magistrales, imprégnées des caractères hellénistiques, parachèvent un programme urbanistique et monumental cohérent (forum, temple, théâtre), elles définissent les villes qu'elles entourent, à la manière d'un péribole (d'où l'exagération de leur parcours), comme des lieux consacrés à la dévotion d'Auguste et de Rome. Par la suite, après Auguste, comment expliquer la raréfaction de ces enceintes si ce n'est justement par le développement du culte impérial? L'absence d'une enceinte à Lyon, dépositaire du sanctuaire confédéral des Trois Gaules, ne pourrait-elle se comprendre ainsi? Narbonne, même si elle fut protégée par quelques fortifications à ses origines, ne posséda pas d'enceinte. Concernant ces deux villes, la réponse peut ètre plus complète ou tout aussi bien différente: leur statut de capitale provinciale qui les élève d'emblée au-dessus des autres cités les autorise à ne pas convoiter cette "gloire encombrante» (Jullian, 1920, V, p. 50) dont même Rome se dispense et que réclame ailleurs la municipalis aemulatio (Gros, 1987, p. 163-164). 
N.B. - Ce travail doit beaucoup à la perspicacité de $M^{\text {mes }}$ A. France et $\mathrm{S}$. Reverdy qui furent chargées sur le site de la lourde responsabilité de la fouille des zones attenantes à l'enceinte et au sens d'observation des architectes: MM. A. Fauré qui exécuta tous les relevés du monument et D. Bruneau qui réalisa les études axonométriques. Les mises au net des documents graphiques et les figures 10, 17, 18 ont été effectuées par Mme L. de Messey.

Sont également vivement remerciés M. F. Dieulafait, numismate, qui étudia toutes les monnaies découvertes sur le site, et MM. A. Vernhet et T. Martin, respectivement spécialistes des productions des ateliers de La Graufesenque et de Montans, qui identifièrent les céramiques sigillées.

Toutes les photographies sont de l'auteur, sauf la figure 11 due à $\mathrm{P}$. Terrancle, photographe.

Enfin, je voudrais exprimer toute ma gratitude à $\mathbf{M}$. R. Lequément, Directeur des Antiquités historiques de MidiPyrénées à l'époque de l'opération Larrey, et à M $M^{\text {me }} \mathrm{L}$. de Messey pour leur soutien permanent tout au long de l'entreprise.

\section{BIBLIOGRAPHIE}

Adam J.-P.

1981 : L'archilecture militaire grecque, Paris.

$1984:$ La construction romaine. Materiaux et techniques, Paris, Picard, 368 p., 746 fig.

\section{Baccrabère G.}

1974: Le rempart antique de l'institut catholique de Toulouse, Chronique 4, Supplément au Bulletin de Littérature Ecclésiastique, Toulouse.

1977 : Élude de Toulouse Romaine, Chronique 3, Supplément au Bulletin de Liltérature Ecclésiastique, Toulouse.

\section{Berthe V.}

1987 : L'enceinte gallo-romaine de Toulouse, Mémoire de mầtrise, Univ. de Toulouse Le Mirail, dactyl.

\section{Catalo $\mathbf{J}$.}

1990 : Le bâtiment médiéval du site de l'ancien Ilòpital Larrey (Toulouse), in: Archéologie et vie quolidienne aux $\mathrm{XIII}^{\mathrm{e}}$ $X I V^{e}$ siècles en Midi-Pyrénées, Musée des Augustins, Toulouse, p. 72-75.

\section{Cazes D.}

1988 : Le théâtre romain de Tolosa, in: Palladia Tolosa, Toulouse romaine, Musée Saint-Raymond, Toulouse, p. 81-83.

\section{Coarelli F., La Regina A.}

1984 : Abruzzo, Molise, Guide archeologiche Laterza, 9, RomaBari.

\section{Filippo R. de}

1990 : Les fosses-dépotoirs, La céramique de Saintonge, in:

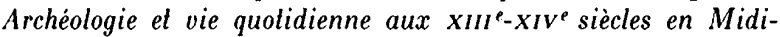
Pyrénées, Musée des Augustins, Toulouse, p. 139-140 et 339340.

\section{Février P.-A.}

1969: Enceinte et colonie (de Nîmes à Vérone, Toulouse et Tipasa), Revue d'Études Ligures, 35, p. 277-286.

\section{Garlan Y.}

1974 : Recherches de Poliorcétique Grecque, Bibliothèque des Écoles Françaises d'Athènes et de Rome, 223 (cet ouvrage contient le traité de poliorcétique de Philon de Byzance, traduit et commenté par l'auteur).

\section{Goudineau Chr.}

1980 : La ville antique, in : Dury G. (dir.), IIistoire de la France urbaine, 1, Paris, Seuil, 600 p, 452 fig.

1990 : César et la Gaule, Paris, Ed. Errance, 365 p.

1991 : Introduction, in : Les villes augustéennes de Gaule, Actes du Colloque international d'Autun, juin 1985, Autun, p. 7-15.

\section{Goulpeau L.}

1990 : Compte rendu de l'étude archéomagnétique de la datation des structures du sile de Toulouse, Hopital Larrey, Univ. de Rennes, Département de physique cristalline, Laboratoire d'archéométrie, dactyl., 12 p., 9 fig.

\section{Grama J. P.,}

1986 : Rapport pour une inscriplion à l'inventaire supplémentaire de cerlains éléments du sile de Larrey, Toulouse, dactyl.

\section{Grenier A.}

1931: Manuel d'archéologie gallo-romaine, I, Paris, Picard, 619 p., 232 fig., 2 cartes.

1958 : Manuel d'archéologie gallo-romaine, III, 1, Paris, Picard, 560 p., 187 fig.

Gros P.

1978 : Archilecture et Sociëtè à Rome et en Italie centro-méridionale aux deux derniers siècles de la République, Latomus, 156 , $101 \mathrm{p} ., 22 \mathrm{pl}$.

1987 : Rapport de synthèse, in : Les enceintes augustéennes ..., p. 159-164.

1991 : La France gallo-romaine, Paris.

Jullian C.

1920 : Hisloire de la Gaule, V, Paris.

\section{Labrousse $\mathrm{M}$.}

1968 : Toulouse antique, des origines à l'établissement des Wisigoths, Paris.

1971 : Les fouilles de la Place du Capitole, Bulletin de la Socièté Archéologique du Midi de la France, 1969-1974, IV, 4 s., p. 189-199.

Le Bot-Helly A.

1987 : L'enceinte de Vienne, in : Les enceintes augusteennes ..., p. 51-61. 
Le Gall J., Le Glay M.

1987 : L'Empire Romain, Paris.

Les enceintes augustéennes...

1987: Les enceintes augustéennes dans l'Occident romain (France, Italie, Espagne, Afrique du Nord), Actes du colloque international de Nîmes, II Ie Congrès archéologique de la Gaule méridionale, 9-12 oct. 1985, École Antique de Nîmes, 18, nouv. sér., 166 p., 58 fig.

\section{Lugli $\mathbf{G}$.}

1957 : Tecnica edilizia romana, con particolare riguardo a Roma e Lazio, Roma.

Magdinier A.-G., Thollard P.

1987 : L'enceinte romaine d'Orange, in: Les enceintes augusléennes ..., p. 77-96.

\section{Mansuelli G.-A}

1971 : Urbanistica e architettura della Cisalpina romana fino al III sec. e. n., Latomus, 111, 228 p., $105 \mathrm{pl}$.

Martin R.

1965 : L'Urbanisme dans la Grèce Antique, Paris.

Pailler J.-M.

1988 : Domitien et "La cité de Pallas». Un tournant dans l'histoire de Toulouse Antique, Pallas, Revue d'Éludes Antiques, XXXIV, p. 99-109.

\section{Palladia Tolosa}

1988 : Palladia Tolosa. Toulouse romaine, Catalogue d'exposition, Musée Saint-Raymond, Toulouse, $191 \mathrm{p}$.

Pasqualini M.

1992 : Fréjus, La Plate-forme, in : Annuaire des opérations de terrain en milieu urbain, 1991, Centre national d'archéologie urbaine, Tours, p. 29, note 111.

\section{Porra V.}

1990: Une occupation de la période de transition Bronze Final/I I Age du Fer à l'ancien hôpital Larrey (Toulouse), DEA, École des Hautes Études en Sciences Sociales, Toulouse, dactyl.

\section{Rebecchi F.}

1987 : Les Enceintes Augustéennes en Italie, in : Les enceintes augustéennes ..., p. 129-150.

\section{Reverdy S.}

1990 : Verreries à l'ancien Hôpital Larrey (Toulouse), in : Archéologie et vie quotidienne aux $\mathrm{XIIr}^{e}-\mathrm{XIV}^{e}$ siècles en MidiPyrénées, Musée des Augustins, Toulouse, p. 186-191.

Rico Chr

1988: La brique à Toulouse dans l'Antiquitè, in: Palladia Tolosa, Toulouse romaine, Musée Saint-Raymond, Toulouse, p. 84-87.

Rivals Cl., Camboulives R., Angely G.

1972 : Civitas Tholosa, Toulouse d'après les plans anciens, Toulouse.

\section{Soutou A.}

1969 : Un secteur méconnu de l'enceinte gallo-romaine de Toulouse, OGAM, XXI, p. 135-154.

Vidal M.

1988 : Les temples, in: Palladia Tolosa, Toulouse romaine, Musée Saint-Raymond, Toulouse, p. 12-13. 